\title{
Ontology-Based Context Modeling in Service-Oriented Computing: A Systematic Mapping
}

\author{
Oscar Cabrera*, Xavier Franch, Jordi Marco \\ Universitat Politècnica de Catalunya - BarcelonaTech, ESSI - UPC. \\ 08034 Barcelona, c/Jordi Girona 1-3, Spain \\ \{ocabrera, franch\}@essi.upc.edu,jmarco@cs.upc.edu
}

* Corresponding author. Address: Universitat Politècnica de Catalunya - BarcelonaTech, ESSI - UPC. 08034 Barcelona, c/Jordi Girona 1-3, Omega Building S-208, Spain.

e-mail: ocabrera@essi.upc.edu

\begin{abstract}
Context: Service-oriented computing and context-aware computing are two consolidated paradigms that are changing the way of providing and consuming software services. Whilst service-oriented computing is based on service-oriented architectures for providing flexible software services, context-aware computing articulates different phases of a context life cycle for changing the behavior of such services. The synergy between both paradigms provides the context to this study.

Objective: This study analyzes the current state of the art of context models, specifically: (1) which are these proposals and how are they related; (2) what are their structural characteristics; (3) what context information is the most addressed; and (4) what are their most consolidated definitions. Given their dominance on the field, the study focuses on ontology-based approaches.

Method: We conducted a systematic mapping by establishing a review protocol that integrates automatic and manual searches from different sources. We applied a rigorous method to elicit the keywords from the research questions and selection criteria to retrieve the papers to evaluate.

Results: Overall, 138 primary studies were selected to answer our research questions. These proposals were studied in depth by analyzing: 1) distribution along time and their relationships; 2) size correlated with the number of classes and levels of the context model, and coverage of the definitions provided as indicator of quality provided; 3) most addressed context information; 4) most consolidated definitions of context information.

Conclusions: The contribution of this survey is to make available a unified and consolidated body of knowledge on context for service-oriented computing that could be instantiated and used as starting point in a variety of use cases. This sweeping view on the anatomy of context models may help avoiding the postulation of new proposals not aligned with the current research.
\end{abstract}

\section{Keywords}

Context-aware computing; context modeling; ontology; service-oriented computing; systematic mapping.

\section{Introduction}

From the perspective of service-oriented computing, context-aware computing involves the management of context within different essential phases of the context life cycle that show how the context data moves from phase to phase in software services. According to Hong et al. "to provide adequate service for the users, applications and services should be aware of their contexts and automatically adapt to their changing contexts-known as context-awareness" [1]. Similarly, Coutaz et al. state that "context is key in the development of new services that will impact social inclusion for the emerging information society" [2]. In this sense, context-aware computing is critical in services that support different tasks of every day's life. 
From a data and knowledge engineering perspective, the context life cycle, as the core of context-aware computing is divided into four stages: 1) context acquisition, responsible for monitoring the context from different sources; 2) context modeling, in charge of modelling and representing the data acquired in meaningful context information; 3) context reasoning, which derives high-level context information from low-level context raw data; 4) context distribution, which exploits high-level and low-level context in context-aware software services [3]. In this paper, we are interested in context modeling, which represents an expanding research field and relevant issue into the context-aware computing and plays an important role into the context life cycle. For instance, in the context-aware software development process, it plays an important role for defining the core data that could be processed in certain application (e.g., data for deducing the location of a person), the data schema that should be managed in the repositories, etc.

The problem that motivates this study is the lack of a common body of context knowledge easy to reuse, consistent, mature, providing the primitives (concepts and properties) needed to model different aspects of an entity (e.g., services). On the contrary, different ontology-based context modelling approaches have emerged to structure the context knowledge with lacks of completeness, stability and abstraction to be more reusable. In fact, as it happens in many other areas, it does not exist a universally accepted standard or approach agreed by the scientific community; instead, several proposals have been presented for specific or general purposes. These proposals may diverge in several matters: facets addressed, ontology size and completeness, structure and terminology, underlying principles, semantic factors, and even in the formalization degree for knowledge representation: basic classification (hierarchy or taxonomy), highexpressiveness (ontology), ... Therefore, it may be argued that it is necessary an effort to assess the current state of the art of ontology-based context modeling focused on analyzing and evaluating the context knowledge pieces and engineering artifacts proposed.

The aim of this study is to identify and relate existing proposals, assess them with respect to some criteria and conclude with the most consolidated context knowledge as well as the most remarkable gaps to bridge. Particularly, the study is focused on the analysis of the current state of the art of context modeling delimited by 1) ontology-based context modeling, since ontologies are the most used conceptual artefact to represent context mainly due to their reasoning capabilities; 2) those models representing a basic form of an ontology such as hierarchies or taxonomies; 3) abstract or domain specific context ontologies; 4) ontologies characterizing some aspect of a service. For this purpose, we have conducted a systematic mapping (SM) according to the guidelines described in [4] and considering good practices from [5] to accurately retrieve and analyze different context models by defining and performing a review protocol. The SM comprises three phases: planning the review, conducting the review, and reporting the review.

As a result of such review, we have been able to identify the strengths and weaknesses of the state of the art. The importance of this work lies mainly along two lines, namely providing an overview of existing ontology-based context models and making available a consolidated context knowledge easy to reuse, specifically in current areas moving towards the innovation and deployment of new services such as Smart Cities, the Internet of Things and Self-Adaptive Systems. Particularly, different benefits can arise from this study, among others: compiling the features that should contain a consistent context ontology; reporting the reasons for increasing the productivity and decreasing the time of a developer of context-aware applications; providing an accessible and integrated overview of relevant context knowledge modelled to characterize services; avoiding misunderstandings by compiling semantic and conceptual ambiguities of the proposed ontologies; increasing the appropriate modelling of prospective contributions by exposing best practices and design patterns of existing models; avoiding model redefinitions to reduce costs. 
The structure of this work is organized as follows. Section 2 presents an introductory background of the main streamlines that influence our work. Section 3 addresses the activities carried out in the first two stages of the SM comprising planning and conducting the review. Section 4 reports the results generated in the review. Section 5 discusses the findings of this study. Then, Section 6 reviews the validity threats that could have occurred during the review. Finally, Section 7 concludes the paper.

\section{Background}

\subsection{Definitions of context for services}

Context is a broad concept and several definitions are provided in the academic literature. Bazire and Brézillon presented an attempt to point out some problematic issues about the understanding of context noting that its definition varies depending on the study field, with more than 100 definitions that were analyzed [6]. For the study field of this paper, we adopt the widespread definition given by Dey: "Context is any information that can be used to characterize the situation of an entity. An entity is a person, place, or object that is considered relevant to the interaction between a user and an application, including the user and applications themselves" [7]. We consider that this definition is generic enough as to be suitable to extract different information of a context model, such as:

- To understand what a context is and how it can be effectively applied.

- To identify primitives (concepts and relations) modelled in an existing context model. For instance, context information and entities relevant in existing interaction process with a service. According to Badidi and Taleb [8], a context is useful to identify various situational circumstances from the viewpoint of services (e.g., identity of the client who invoked the service, location and time at which the client invokes the service, etc.).

- To identify different conceptual patterns presented in a proposal.

- To identify different conceptualizations and specifications of context in a model.

\subsection{Context modeling research, perspectives and formalisms}

According to Henricksen [9] "a context model identifies a concrete subset of the context that is realistically attainable from sensors, applications and users and able to be exploited in the execution of the task. The context model that is employed by a given context-aware application is usually explicitly specified by the application developer, but may evolve over time". Context modeling should provide a well-defined structure of context information facilitating the activities carried out in all other context life cycle stages [3]. When the model does not allow representing relations and dependencies, it is difficult to carry out reasoning and inference to generate further context information classes. Furthermore, the raw data acquired from sensors must be translated into context information classes to give sense and structure. In this regard, context modeling is an effective method of gathering, representing and sharing context information across different information systems [10].

Context information represents the basic element in a context model adopting atomic items that can be classified based on different categorization and formalization schemes following a systematic methodology. As stated by Crowley et al., context is an information space that can be modeled as a directed state graph, where each node denotes a context, and edges denote the conditions for change in context [11]. Each context is defined by a set of entities (including literal values, as well as real-world and information objects), a set of roles (for example, functions) that entities may satisfy, a set of relations between the entities, and a set of situations. Entities, roles and relations are modeled as expressions over observables captured and inferred by the system at the appropriate level of abstraction. A well-defined context model will minimize the complexity of services, enhancing their maintainability and ability to evolve [12]. 
Different approaches to model context can be found in the literature. Recently, Perera et al. presented a comparison of the six most popular context modeling categories of techniques, namely key-value, mark-up scheme, graphical, object oriented, logic-based and ontology-based [3]. Based on the analysis and considering previous works in the topic, the authors concluded that the most appropriate technique to manage context is ontology-based modeling.

According to Noy and McGuiness, an ontology is a formal explicit description of a domain, consisting of classes, which are the concepts found in the domain, also called entities [13]. Representing context information classes through ontologies is a challenge in the sense of describing in a precise and traceable manner this information. Ontologies seem to be the way to represent the knowledge concerning context in several domains. As stated by Noy [14] and Chen et al. [15], ontologies are believed to be a key feature in the making of context-aware distributed systems due to the following reasons:

- Ontologies enable the sharing of knowledge by open dynamic agents (e.g. web services).

- Ontologies supply semantics for intelligent agents to reason about context info.

- Ontologies promote the interoperability among devices and computational agents.

Other authors as Sudhana et al. mention further advantages of ontology for context modeling [16]:

- The amount of information to be delivered to the user can be reduced based on learner preferences and context ontology.

- The user needs and expectations can be predicted to recommend suitable material based on the ontological inference mechanism.

- It enables formal analysis of domain knowledge for context reasoning from the explicitly defining context ontology.

- The main purpose of ontology-based context model is to enable semantic interoperability and to provide common understanding of the structure of context information classes among users.

For all these reasons, this SM is focused on context modelling from the perspective of ontologies, including their basic structure, namely taxonomies and hierarchies.

\section{Planning the systematic mapping}

\subsection{Identification of the need for a review}

Petersen et al. [4] highlight the importance of motivating the need and relevance of any SM. For this purpose, we followed the recommendations given by Kitchenham and Charters [5] since they provide more evidence at this stage. Hence, researchers are required to seek and identify existing works related with the subject and assess them with respect to some criteria. Therefore, we searched for other reviews and assessed them by considering the relevant aspects of this review in contrast with the existing contributions to ensure and confirm that a new one is necessary. In order to expand the number of results retrieved, besides searching systematic literature studies, we also sought other studies published in scientific venues such as state of the art contributions, surveys, etc. To do so, we defined an additional search protocol, similar to the main search in the SM, to recognize such other reviews.

Therefore, we applied basically the protocol that will be explained in detail in the rest of the section, just adding to the search keywords the following terms: "state of the art", "survey", "review", "SLR" and "systematic mapping". As a result of applying automatic and manual searches, we found 16 papers fulfilling the search criteria (the full list is available online [17], Annex A). However, after inspecting them, we found that although these studies provide strong contributions in the fields of context and context-awareness they are oriented to other purposes that did not affect the objectives of this review (see comparative table in online Annex B at [17]). 
Only Bauer [18] made an effort to provide a 3-level hierarchy of context information classes but: she considered a very limited amount of context models; semantic issues were not considered; reusability resources were not analyzed. Some remainder works focused on evaluating context models and their applications, while others were centered on context-awareness analyzing and evaluating how the stages of the context life cycle are managed. Consequently, we confirmed the need to consolidate existing research on context modelling based on ontologies from a service-oriented computing perspective.

\subsection{Research questions}

To formulate the research questions, we used the goal of the review [4] and the PICO (Population, Intervention, Comparison and Outcome) criteria [5]. Using PICO, research questions are defined by means of these criteria allowing dissecting useful keywords to structure the main search string in order to obtain more evidence from the study field.

The goal of this SM is to assess the context knowledge pieces defined in existing context models based on ontologies to identify their reusability in proposals from the perspective of service-oriented computing. In this context, the comparison criterion is out of the scope of this review since we are not interested on comparing context models. Therefore, the main research question was finally formulated as shown in Table 1. As it can be seen, the main research question was highly generic, so that we refined it into specific subquestions considering interests and motivations of the review.

Table 1: Research questions

\begin{tabular}{|c|c|}
\hline \multicolumn{2}{|c|}{ Main research question } \\
\hline \multicolumn{2}{|c|}{$\begin{array}{l}\text { In the field of services }(\mathrm{P}) \text {, do ontology-based context models }(\mathrm{I}) \text { proposed so far provide an adequate and structured } \\
\text { set of context knowledge pieces to be reused in prospective proposals that can define their context }(\mathrm{O}) \text { ? }\end{array}$} \\
\hline \multicolumn{2}{|c|}{ Specific sub-questions } \\
\hline Research sub-question & Interest and motivation \\
\hline $\begin{array}{l}\boldsymbol{R Q 1 . 1 .} \text { What is the chronological overview of } \\
\text { the research done so far in ontology-based } \\
\text { context models? }\end{array}$ & $\begin{array}{l}\text { Identify the proposals in the field, find their interrelationships } \\
\text { and distribute them along time to find any significant trend, } \\
\text { considering also their provenance (academy or industry) }\end{array}$ \\
\hline $\begin{array}{l}\boldsymbol{R Q 1 . 2 .} \text { What are the characteristics of the } \\
\text { proposed ontology-based context models? }\end{array}$ & $\begin{array}{l}\text { Make explicit the main characteristics of these context models in } \\
\text { terms of size, structure and completeness }\end{array}$ \\
\hline $\begin{array}{l}\boldsymbol{R Q 1 . 3 . ~ W h i c h ~ c l a s s e s ~ o f ~ c o n t e x t ~ i n f o r m a t i o n ~} \\
\text { and entities are the most addressed in } \\
\text { ontology-based context models? }\end{array}$ & $\begin{array}{l}\text { Identify aspects related to the scope of these context models, such } \\
\text { as: what are the contexts and entities attracting more attention } \\
\text { from researchers, since it may help to understand their priorities } \\
\text { and eventually some research gaps }\end{array}$ \\
\hline $\begin{array}{l}\boldsymbol{R Q 1 . 4 . ~ W h a t ~ a r e ~ t h e ~ m o s t ~ c o n s o l i d a t e d ~} \\
\text { classes of context information and entities in } \\
\text { ontology-based context models? }\end{array}$ & $\begin{array}{l}\text { We aim at identifying the most recurrent definitions of classes of } \\
\text { context information and entities, which in some sense could be } \\
\text { considered as the starting point of any new future proposal }\end{array}$ \\
\hline
\end{tabular}

\subsection{The Protocol}

\subsubsection{Bibliographic sources}

The search process conducted in this SM comprised automatic and manual searches. The automatic search was carried out by using several digital libraries (DLs), and the manual search through collecting the works from specific journals and conferences of the field of interest. Advantages and drawbacks of both approaches were analyzed through a case study by Kitchenham et al. [19]. Based on this analysis, we decided to integrate both strategies by accomplishing an automatic search in the selected DLs and complementing the results with manual searches to the most relevant conferences and journals in case that some conference edition or journal issue was missing in such DLs. To select the bibliographic sources 
relevant to this review, we followed the study and selection criteria provided by Dieste et al. [20]. As a result, the selected DLs were Scopus, IEEE Xplore and ACM Digital Library.

Concerning manual searches, we identified a list of journals and conferences relevant to this study. We targeted venues mainly in the field of software services and context modeling such as information systems and technologies, pervasive and ubiquitous computing, human-computer interaction, modeling and data engineering, and semantic web (see the full list in online Annex C, Table 1 at [17]). These venues were selected from the top-ranked list based on the JCR impact factor for journals and the CORE-A status ${ }^{1}$ for conferences. It is important to keep in mind that these sources were identified with the purpose of checking completeness of the study, but the main bibliographic corpus came from the automatic searches, due the broad spectrum of the selected DLs.

Conferences and journals shown in Annex C [17] were reviewed to verify that all their editions from 2001 to 2015 were indexed and published in the selected DLs. The period from 2001 to 2015 was selected as starting point since it is possible to identify a consolidation during this period of current research topics on context and services such as Internet of Things, *-as-service and Service Oriented Computing. This verification allowed us to identify when to apply automatic or manual searches, i.e. when the journal or conference was published with all its editions in some of the selected databases means that it is sufficient to apply an automatic search, otherwise, it is necessary to apply manual searches for each missing edition, leading to searches in a specific DL of a conference or journal. The results are presented in Annex D at [17]. To sum up, 7 journals and 17 conferences required manual searches because at least one edition was missing in all the DLs used.

\subsubsection{Keywords used}

To compose the main search string, we acquired the keywords from the PICO criteria specified in Section 3.2. Specifically, Population and Intervention criteria are used to extract them. In the context of this study Comparison was discarded in the establishment of the research questions (Section 3.2) and Outcome was not considered because it is not based on a particular measurement in the research questions. As stated by Petersen et al. [4][21] and Kitchenham et al. [22], the Outcome criterion is not always applicable. Hence, from each term of the Population and Intervention criteria, we identified the keywords used to build the search string as depicted in Table 2.

Table 2: Keywords and search string

\begin{tabular}{|c|c|c|}
\hline Criteria & Keyword & Variants \\
\hline Population & Service & "service", "services" \\
\hline Intervention & $\begin{array}{l}\text { Context } \\
\text { model }\end{array}$ & $\begin{array}{l}\text { "context model", "context models", "contexts model", "contexts models"; } \\
\text { "context ontology", "context ontologies", "contexts ontology", "contexts } \\
\text { ontologies"; "context taxonomy", "context taxonomies", "contexts } \\
\text { taxonomy", "contexts taxonomies"; "context hierarchy", "context } \\
\text { hierarchies", "contexts hierarchy", "contexts hierarchies" }\end{array}$ \\
\hline \multicolumn{3}{|r|}{ Search string } \\
\hline \multicolumn{3}{|c|}{ 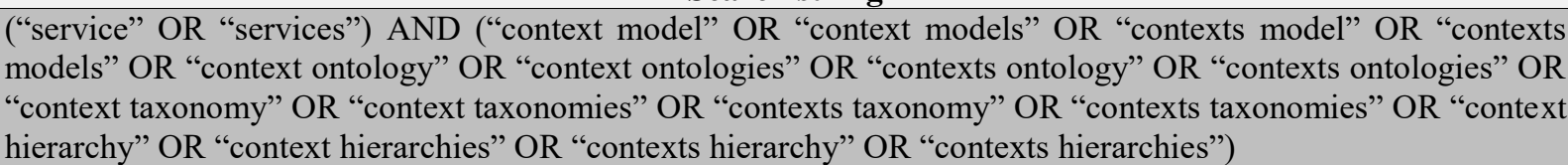 } \\
\hline
\end{tabular}

\footnotetext{
${ }^{1}$ There is no standard procedure to select the list of conferences. Although the CORE-A index, as any other, can raise controversy, we consider it as a good indicator for our purposes. See http://core.edu.au/index.php/conference-rankings.
} 
Note that to build the search string, variants inside Population and Intervention are interconnected through OR connectors (e.g. "service" OR "services") and finally, these criteria are joined through an AND connector. Since we are interested in ontology-based approaches, we added "context ontology" to the search string. Furthermore, according to Fernández-López et al., there are different levels to express an ontology referring to taxonomies or hierarchies [23], therefore we added also these terms. Last, in order to increase the quality and to improve the searches, we configured and simplified singular and plural forms of the search string by using the guidelines provided in each of the databases selected due to known limitations (e.g., length of the query in some databases). Such search string configurations for each database selected are specified in Annex E at [17].

\subsubsection{Selection criteria}

Once the bibliographic sources and the search string were specified, the search was conducted by title, abstract and keywords both in works obtained automatically or manually. After retrieving the results, we followed several steps to filter the candidates:

- Filter by title. Step to quickly identify and remove noise from results. After this selection, documents whose scope was clearly unrelated to context models were removed.

- Filter by abstract. Step used to delete works that although being related to context models, did not present an ontology-based context model as a contribution of the paper.

- Filter by fast reading of full paper. Step to discard those papers that did not fulfil properly the following inclusion criteria: (1) defining explicitly the context model; (2) presenting a context model that can be applied from the perspective of the service-oriented computing, i.e., the model conceptualizes a process or some of the entities involved in the service provisioning and consumption (e.g. user or provider of a service, service composition, etc.).

- Snowballing. Step that gathers further works during the SM process. To conduct this step, we employed backward snowballing that identifies relevant works from the reference list of the articles. We have included those referenced works that fulfil the previous inclusion criteria.

The results obtained from the searches are described as follows (see Figure 1): 787 papers automatically from Scopus, 293 from IEEE Xplore and 72 from ACM DL yielding a total of 1.152 papers from which 220 were removed as they were duplicated, resulting 932 papers found automatically. Then, 111 papers were added from manual searches in selected venues (as explained in Section 3.3.1), obtaining 1.043 papers from which 712 were deleted by title and abstract, resulting in 331 papers to filter by fast reading. We discarded 17 papers that were not available through our University resources and whose authors didn't provide a copy under our request. Finally, the resulting papers after filtering by full paper were 145 and after adding 19 papers by snowballing, we obtained 164 papers to include in the SM. Afterwards, when analysing them in detail, we found a set of equivalent proposals, i.e., proposals from the same authors with a similar contribution; in this case we selected the most complete proposal to be evaluated and reviewed all of them to identify relevant information not considered in the chosen representative papers. The list of papers along the selection process is available in Annex $\mathrm{F}$ and the final reference list of the 138 assessed proposals in Annex G at [17].

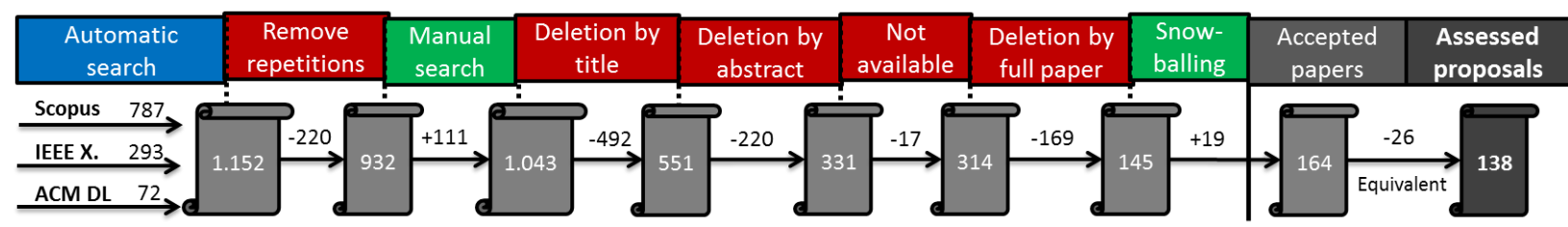

Figure 1: Primary studies selection process 


\section{Results of the review}

In this section, the research questions specified in Section 3.2 are addressed. The results are an abstraction of the most important information retrieved by complete readings performed on each of the contributions selected. An extended report of the analyzed information (data extraction) from the proposals is provided at [17].

\subsection{RQ1.1. What is the chronological overview of the research done so far in ontology-based context} models?

\subsubsection{Analysis per year}

Figure 2 displays a bar chart specifying papers discovered and selected in the review process by year of search comprising a period from 2001 to 2015. Since this analysis is focused on publications by year, in this research question we considered the 164 accepted papers instead of the 138 finally assessed, i.e., before discarding 26 equivalent papers (see Section 3.3). As depicted in Figure 2, the amount of papers discovered by applying the search string resembles a bell curve with a peak in 2009. Even considering this decrease, the number of papers discovered in 2015 is still large enough as to consider the topic lively. At its turn, the distribution of the selected papers (which represent only $15 \%$ of the total amount of papers found in the search), presents a double bell curve with peaks in years 2007 and 2014.

The distribution of the accepted papers by countries appears in Annex H [17]. It is not included in the paper for the sake of space.

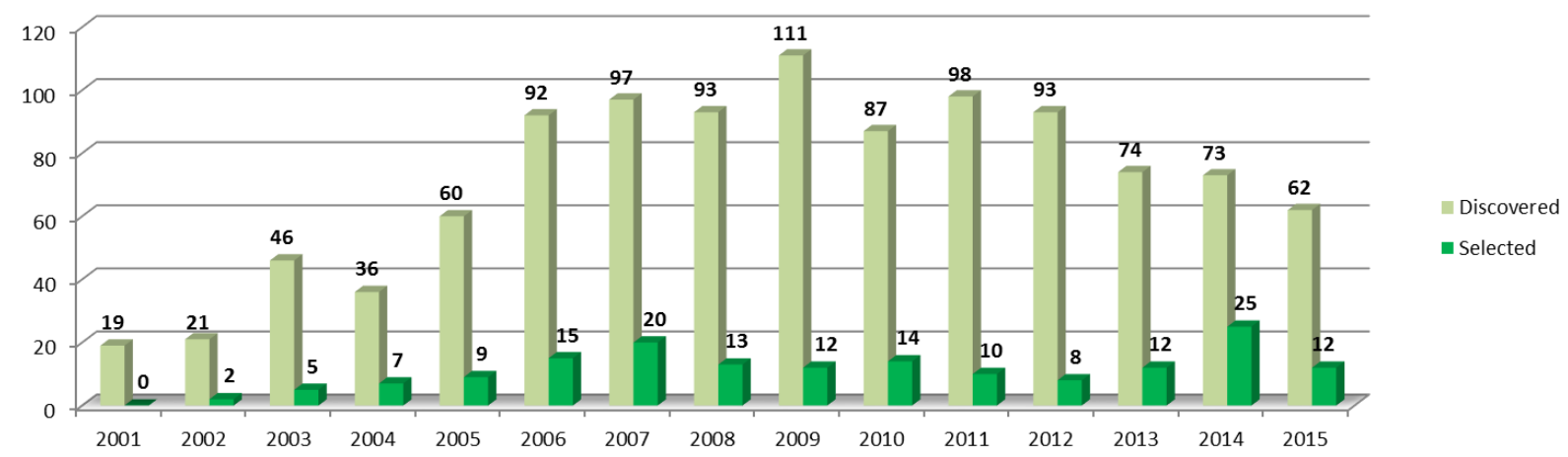

Figure 2: List of papers discovered and selected by year period

\subsubsection{Analysis per provenance}

Figure 3 shows a pie chart describing the percentages of contributions related to university, research centres and industry. As it can be seen, the provenance with largest contributions is linked to universities with 105 $(76 \%)$ of the total amount of contributions in the field, which grows up to $121(88 \%)$ if we consider collaborations. On the other side, proposals solely from industry are the exception: only 3 (2\%), up to 7 $(5 \%)$ if we include industry-university collaborations. It is worth noting that we identified this information based on the affiliation of the authors provided in the selected papers. 


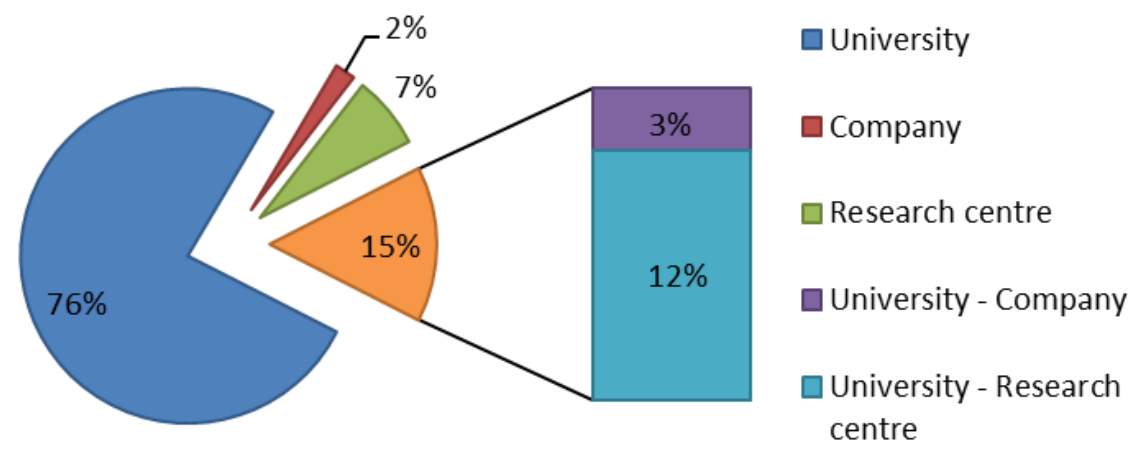

Figure 3: Provenance distribution of selected papers

\subsubsection{Evolutionary analysis}

The goal of this section is to distribute the chronological evolution of the proposed context models for showing their relationships and identifying which ones are the most consolidated, the most influenced and the most influencing proposals. For this goal, we analyzed the chronological evolution of context models and depicted the research done in the field by means of a genealogical tree shown in Figure 4. In the first row of the figure, we have identified different proposals out of the scope of this study, but which represent relevant background of the assessed proposals, regularly cited by them. The contribution by Maslow et al. [24] is the oldest one (1970), and although their proposal was not exactly a context model, it provided suitable vocabulary to be used in context modeling. Similarly, Uschold et al. [25] and the OpenCyc project [26] provided vocabulary from a specific ontology created for other purposes beyond context representation. Other authors with important presence in the field such as Schmidt et al. [27][28], Schilit et al. [29], etc. also exerted influence on the assessed proposals.

Regarding the period of time considered in this study, we found that the first two ontology-based context models proposed were issued in 2002 by Henricksen et al. [M1] ${ }^{2}$ and Held et al. [M2]. During this year, the context models proposed were individual contributions without influences by previous proposals in the field. However, 2003 witnessed the proposal of a first context model [M3] influenced by previous contributions. Since then, some proposals have been developed based on previous models, reusing concepts, vocabulary and definitions. In consequence, most of the oldest context models have been updated or enhanced by other researchers. But also, isolated contributions have been identified with new proposals in the field such as the proposed by Ghannem et al. [M4] and Mok and Min [M5].

Other relevant findings have been classified as follows:

- Standards. During the review of context models, we sought for some standard context model used as starting point of other contributions. However, we did not find such a proper standard. The most similar contribution to a standard is the SOUPA ontology ("Standard Ontology for Ubiquitous and Pervasive Applications") [M6]. This ontology is highly referenced in context research; in fact, as shown in Figure 4, different proposals have been influenced by SOUPA, e.g. Cao et al. [M7], Devaraju and Hoh [M8], Pietschmann et al. [M9], Cadenas et al. [M10], Rubio et al. [M11], Cabrera et al. [M12]. We also noted that several proposals, included SOUPA, reuse a variety of vocabulary from standards developed for other purposes different than context, such as: DAML-Time [30], an ontology of temporal concepts; SUMO [31], an upper ontology that merges several ontologies from distinct domains providing hundreds of terms; OWL-S ${ }^{3}$, semantic mark-up for Web services.

\footnotetext{
${ }^{2}$ References to the primary studies of the SM are written in format [M1]-[M138]. The full list is available in [18], annex G.

${ }^{3}$ http://www.daml.org/services/owl-s/1.0/owl-s.html
} 


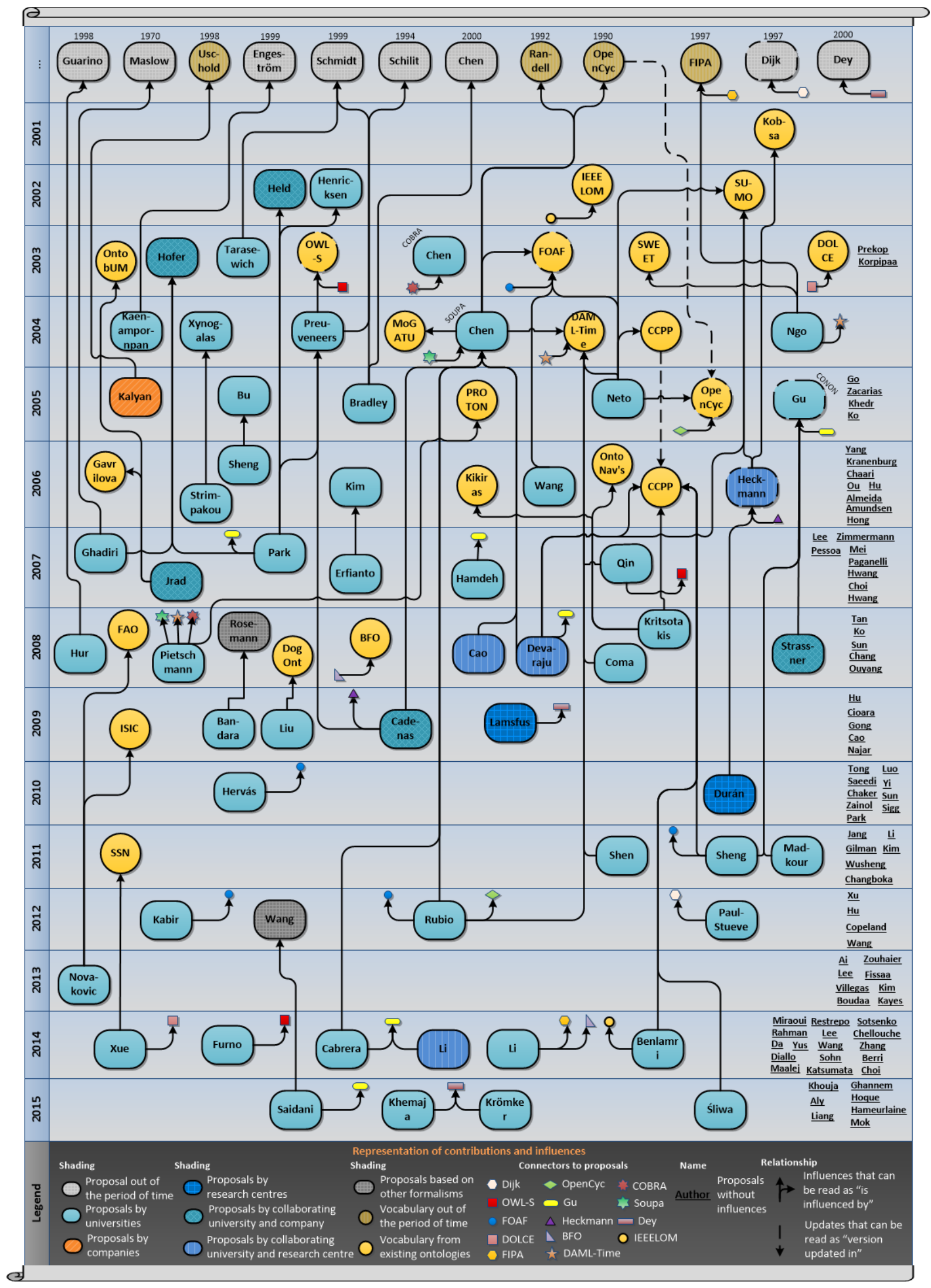

Figure 4: Genealogical tree of ontology-based context models 
- New approaches. There are context models that have not considered previous contributions in their definitions nor have influenced other proposals. These proposals represent 82 of the 138 proposals assessed in the review (59\% over the total), mostly concentrated in the period from 2005 to 2015 with the exception of 2012. The general purpose of these proposals is to provide new approaches or perspectives in context modeling not yet considered in previous contributions. However, although each model provides its own structure and formalism, we found context information classes with the same or similar meaning of another term. To avoid it, the proposals should have reused existing knowledge and provide the resources needed to facilitate future contributions.

- Influences exerted by existing proposals. Contributions that have influenced the definition of other context models represent $8 \%$ of the proposals assessed. As can be seen in Figure 4, the context models with more significant impact in this sense are the oldest ones. Particularly, the most used context models are CONON [M13] and SOUPA [M6] which have been used to develop 9 and 6 context models respectively, followed by Hofer et al. [M14], Preuveneers et al. [M15] and Heckmann [M16] influencing 2 context models each. The remaining contributions proposed by Henricksen et al. [M1], Held et al. [M2], Chen et al. [M17], Xynogalas et al. [M18], Bu et al. [M19], and Kim and Choi [M20] influence 1 context model each. We also analyzed transitivity, namely if a context model $\mathrm{X}$ influences another context model $\mathrm{Y}$ and $\mathrm{Y}$ at its turn, influences another model $\mathrm{Z}$, then we may say that $\mathrm{X}$ also influences $\mathrm{Z}$. The chronology shows that this happened only in a small set of proposals assessed (7\%) such as those influenced by Chen et al. [M6], Preuveneers et al. [M15] and Heckmann [M16].

- Proposals considering different contributions. The context models in this category were developed from three perspectives, namely reusing vocabulary from an existing model with or without context purposes based on ontologies or on other formalisms, adopting the approach of an existing context model or a combination of them. In this regard, contributions considering more models for the development of their own proposals are provided by Chen et al. [M6], Neto and Pimentel [M21] and Park and Kwon [M22] with 5 considered proposals each. Meanwhile Chen et al. and Neto and Pimentel reuse vocabulary from existing ontologies not necessarily context oriented, such as FOAF [32], OpenCyc [26] and DAML-Time [30], Park and Kwon further adopts context model approaches from Henricksen et al. [M1], Held et al. [M2], Hofer et al. [M14], Gu et al. [M13], Preuveneers et al. [M15]. Other proposals considering existing contributions in their models are Devaraju and Hoh [M8], Pietschmann et al. [M9] and Rubio et al. [M11] with 4 proposals each, followed by Cadenas et al. [M10], Preuveneers et al. [M15], Ngo et al. [M23], Bradley and Dunlop [M24], Kritsotakis et al. [M25], and Sheng et al. [M26] with 3 proposals each.

Summing up, the chronological evolution of existing context models shows the need of a standard context model that can be considered as a consolidated basis of context knowledge for new proposals. This lack translates into different variation degrees among the models with respect to conceptualization, semantics in the primitives considered, structural formalizations and design patterns, among others. Although the existing variations can represent research solutions for different domain scenarios, the lack of such a common body of knowledge may represent additional costs (e.g., operation, engineering, maintenance, etc.) in the context-aware software development process. These costs will emerge when developers and modelers are faced to the construction of models from scratch that are not aligned with a common knowledge, postulating again models that cannot be reused or maintained for other developers.

\subsection{RQ1.2. What are the characteristics of the proposed ontology-based context models?}

An ontology-based context model provides structural characteristics and resources that make it suitable for certain purposes. Every proposal of context model should provide the required features to develop models easy to reuse in different situations. According to Fernández-López et al. [23], an ontology, to be reusable, must be well documented during the whole ontology development process and consider definitions of terms already specified whose semantic and implementation is coherent with the terms that are being defined. 
Therefore, to answer this research question, we analyzed and evaluated the structural characteristics and resources of the proposed ontology-based context models in terms of their size and definition coverage addressing completeness issues.

\subsubsection{Size of structural characteristics and resources provided}

According to the background literature on context modeling, a context model should consist of classes and properties, which altogether represent a central knowledge piece that should be defined and documented for increasing its reusability. Therefore, the size that we were interested to analyze and evaluate referred to the amount of context information that classes and properties (datatype and object properties) provide in the selected context models. Concerning object properties, we focused on those belonging to ad hoc binary relations (e.g. isLocatedAt, isAffectedBy, isUsedBy, etc.) since they represent relations between classes of an ontology (e.g. Person isAffectedBy Environment) and could support more powerful semantic analysis and reasoning [23][33]. Relations that belong to the development of basic concept taxonomies such as Subclass-of, Disjoint-Decomposition, Exhaustive-Decomposition, and Partition were not considered in this research question. Such relations that in some extent have to do with hierarchical structure of classes and subclasses are analyzed in Section 4.3.

\section{Number of levels}

The size of the context model was evaluated considering the amount of classes that provide context information and the number of levels of the class hierarchy (which we call depth level). The correlation between the number of classes and the depth level is depicted in Figure 5. We highlight the following:

- From the total amount of classes $(2,756)$ and depth levels (332) in 138 proposals, on average, the proposed context models have 19.97 classes and depth level of 2.4. The largest context models contain more than 40 classes and are arranged into 3 or 4 levels. The largest one by Ngo et al. presents up to 97 classes organized into 4 levels [M23]. The smallest one by Lee and Kwon [M27], Chaker et al. [M28], and Henricksen et al. [M1] present a context model of 3 classes in a single level.

- There are two main regions in the figure. At the bottom, left-most part, 61 models (44.2\% of the total) are expressed in less than 15 classes distributed in at most 2 levels. At the top, right-most part, 26 models $(18.8 \%)$ comprise at least 31 classes organized into 3 or 4 levels.

- Going a bit further in this direction, we observe a linear disposition of the results, which is steeper for few classes and gets flattened as the number of classes increases. Roughly speaking: models with [3, 10] classes tend to be organized in 1 level; models with [7, 18] and [11, 22], in 2 levels; models with $[15,26]$, in 3 levels; and models with more than 27 classes, in 4 levels. The only remarkable exception is the 7 proposals with more than 40 classes arranged in 3 levels.

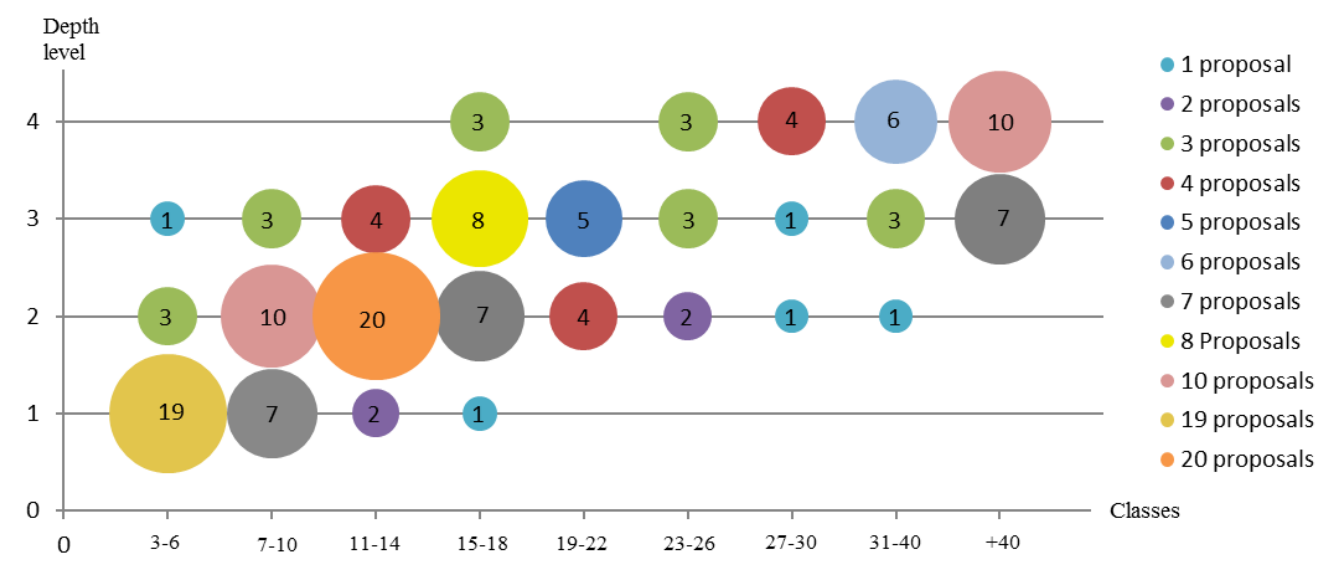

Figure 5: Correlation map between context information classes and hierarchy levels 


\section{Number of datatype properties}

Considering that the proposed context models should specify more details and resources to facilitate their reuse [34], we also evaluated the size of properties (datatype and object properties). Datatype properties link an individual to an XML Schema Datatype value or an RDF literal, i.e., they describe relationships between an individual and data values (e.g. the datatype property "age" links a person with his/her age that is a literal value). Contrary to them, object properties link an individual to an individual or a class to a class (e.g. the object property "hasParent" associates classes such as Person to a Person or in the case of individuals it associates an instantiation of the class Person). Hence, the evaluation was conducted by considering the amount of context information classes relative to the amount of datatype and object properties specified in the proposed context models. This was useful to correlate the quantity of properties provided in a certain number of context information classes.

Figure 6 shows the size of context models regarding datatype properties. We can observe that most of the context models were developed with less than 10 datatype properties (109 proposals out of 138, i.e. $79.0 \%$ ) and in particular, 83 of them $(60.1 \%$ of the total) did not include any. In contrast to them, only 3 contributions (2.1\%) were developed specifying more than 30 datatype properties being the context models proposed by Pietschmann et al. [M9] and Hervás et al. [M29] those providing larger number of datatype properties, 40 properties each.

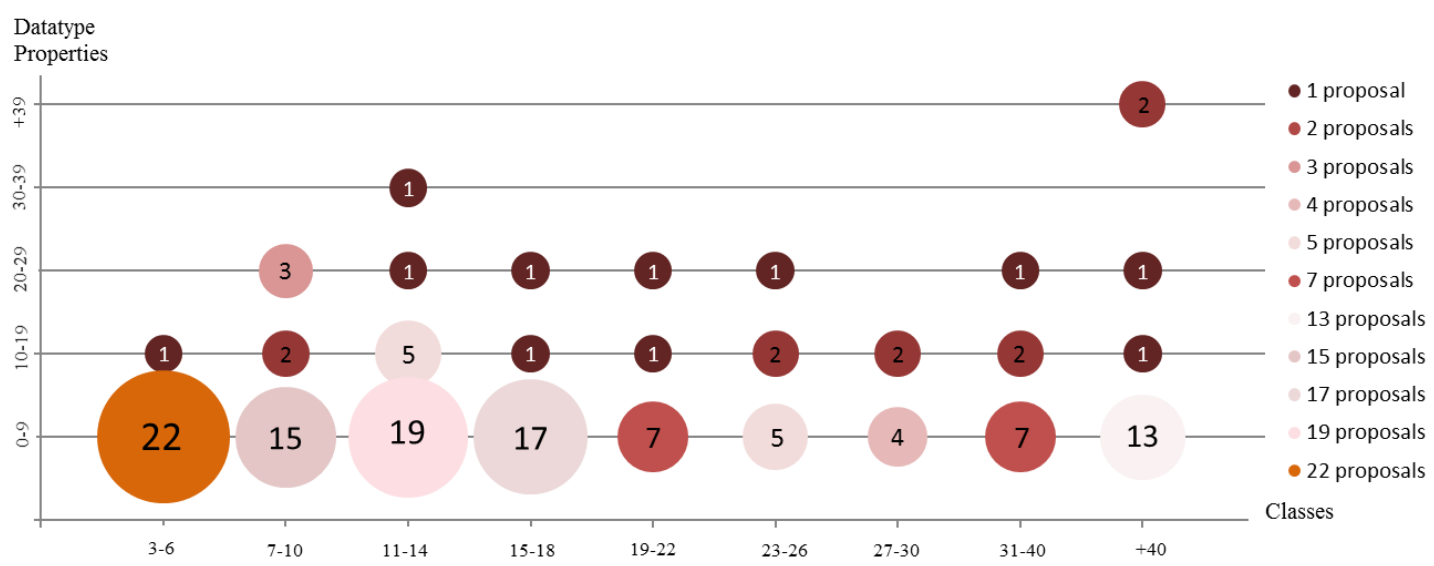

Figure 6: Correlation map between context information classes and datatype properties

As it can be seen, the number of datatype properties is not correlated with the number of classes; in other words, the number of datatype properties does not depend on the number of classes. This was expected since each class of a model can be related to zero or many datatypes. Hence, it is possible to have a model with 5 classes and 5 datatype properties that relate only one of the classes with datatypes (e.g., in a model specifying 3 classes Time, Person and Activity only the Person class was related to different datatype properties such as hasName, hasAge, hasGender, etc. and it was not the case for Time and Activity).

\section{Number of object properties}

The size of object properties that associate classes to classes or individuals to individuals was evaluated by considering the amount of object properties, relative to the amount of classes in a model. The result is summarized in Figure 7:

- The context models with more object properties are provided by Pietschmann et al. [M9] and Hervás et al. [M29] with 56 and 53 object properties linking 67 and 47 classes respectively. On the contrary, the context models with less object properties were identified in 66 proposals $(47.8 \%)$ that do not provide any object property. 
- Figure 7 indicates that most of the context models were developed with a low range of object properties, less than 19 (114 proposals, 82.6\%). From the remaining contributions, only 5 (3.6\%) presented more than 30 object properties.

- Given the data retrieved, there is not a clear correlation between the number of object properties and classes, as also happened with datatype properties.

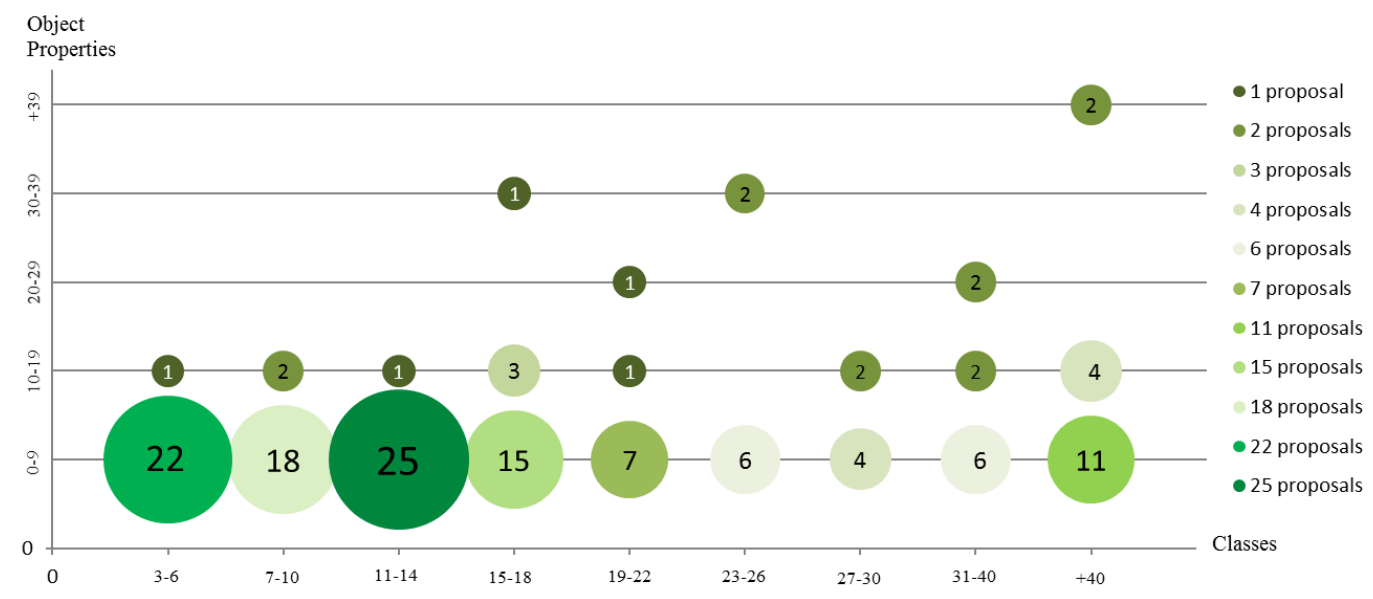

Figure 7: Correlation map between context information classes and object properties

In fact, comparing the two correlation maps depicted in Figure 6 and 7, we can observe that their shape is almost the same. We looked into the details of the proposals and we found a strong overlapping, even if it was not expected since the concepts in the two correlation maps do not depend on each other. In particular, 106 proposals $(77 \%)$ were in the same area of both correlation maps, indicating that these approaches proposed context models that specified datatype properties almost in the same proportion as object properties. As a case worth to mention, from the 22 proposals that appear in the leftmost, bottom area corresponding to 0-9 properties and 3-6 classes, 21 of them are the same in both correlation maps indicating that proposals with more focused or smallest contributions to a context model, are balanced in terms of both types of properties. Similarly, the two proposals in the rightmost, top area are the same for both concepts.

\subsubsection{Definition completeness of structural characteristics and resources provided}

Definition completeness was evaluated on context information classes, datatype and object properties, in order to review the definition coverage of these characteristics, considering that each element in a model should be defined and associated with other resources of the model for better understanding in future reuses. Hence, the completeness evaluation of classes and properties was as follows: 1) for context classes, we evaluated the definitions and semantics provided for each class represented in the context model; 2) for datatype properties, we focused on identifying how many classes were or not related to literal values through datatype properties; 3) for object properties, we identified how many classes were or were not related to another class through object properties. The evaluation specified in 2 and 3 is because in the previous analysis we only identified the size of properties in the context models, but not the number of classes related to a property. It can be useful to identify if all the classes and properties specified in a context model have an active role for inferring or deducing context.

\section{Completeness of context classes}

The pie chart of Figure 8 shows percentages of definition completeness regarding context information classes. As it indicates, only $20 \%$ of the presented proposals ( 28 contributions) have a unique and consistent definition for all the context information classes specified in the corresponding context model, i.e. $100 \%$ definition completeness, either by explicitly defining the context in the paper or by referencing to another bibliographic source that has the definitions. 


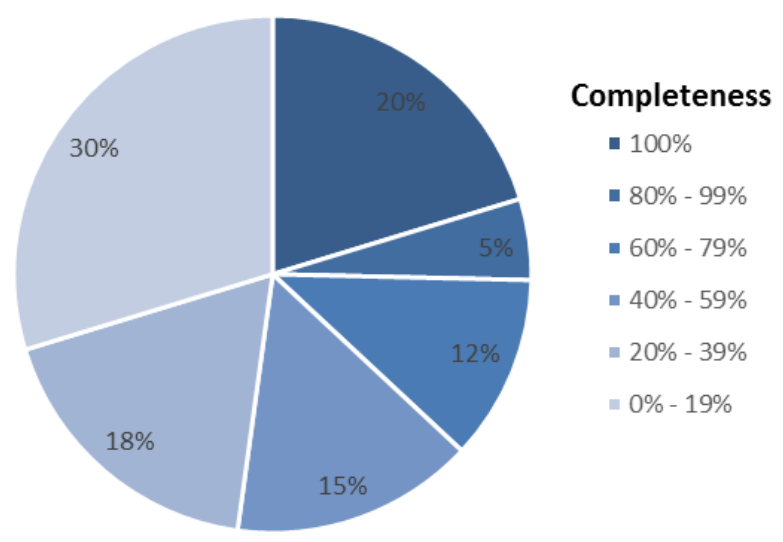

Figure 8: Completeness of context information classes

Proposals with lower percentages of definition completeness present different issues, such as: some context information classes are not defined; some context models are based on other context models and although they are referenced, it is not specified which is the chosen definition for each context information class, leading to different definitions which are not consistent with each other; and the definitions on some context information classes are too vague or ambiguous. Note that as much as $30 \%$ of the proposals (41 contributions) have completeness level below $20 \%$, which clearly is a very bad result. In fact, $20 \%$ of the proposals (27 contributions) do not provide any definition of context information classes.

\section{Completeness of datatype and object properties}

Definition completeness regarding datatype and object properties is illustrated in the pie charts of Figure 9. The findings related to this completeness analysis indicate a percentage of properties that were defined in a model but were not associated with a class of the model (e.g., if a model specifies a size of 10 properties but only 6 are associated with a class, it means that the $40 \%$ of the properties lack of range, i.e. they are defined but not related to any class). Hence, reusing a context model that does not provide $100 \%$ of completeness indicates that a set of properties should be reanalyzed to understand its domain and range of application (e.g., FOAF provides several properties that are not associated nor maintained causing reuse problems).

According to this criterion, the pie chart on the left in Figure 9 indicates that only $4 \%$ of context models (6 proposals) have provided at least a datatype property to relate between $70 \%$ and $100 \%$ of their context information classes with datatypes. The rest has lower percentages and remarkably $81 \%$ of the approaches (112 contributions) do not reach a $20 \%$ level of completeness, pointing out a severe deficiency: most context models do not provide axioms involving datatype properties such as "each instance of the Location class must have an xsd:string data value for the hasCoordinates datatype property".
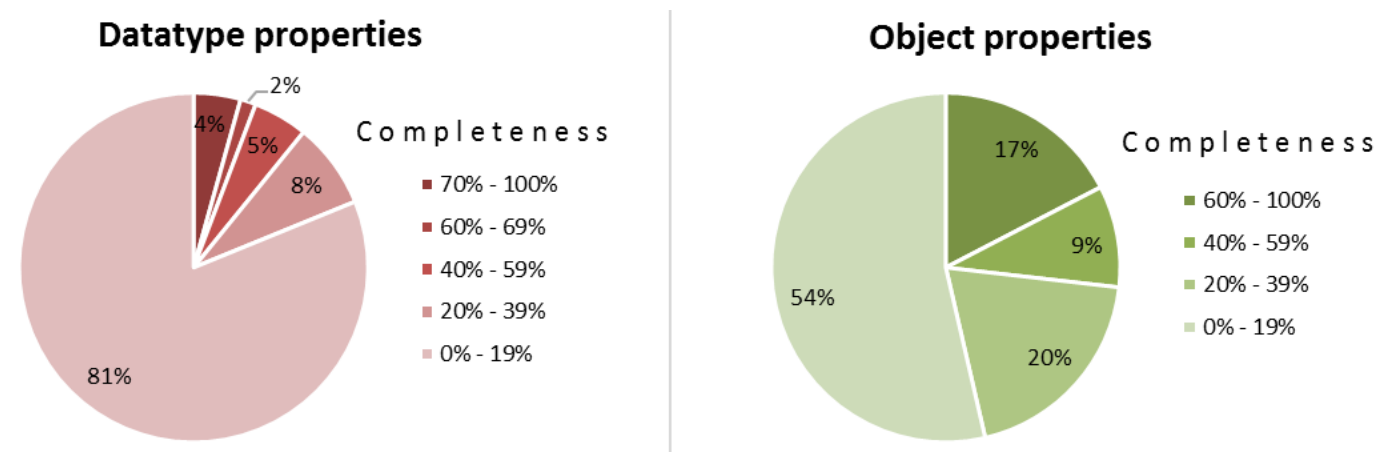

Figure 9: Definition completeness of datatype and object properties 
The pie chart on the right indicates that $17 \%$ of the context models ( 24 contributions) provide between $60 \%$ and $100 \%$ completeness of object properties to associate context information classes, representing a higher proportion than datatype properties but still low considering that the $73 \%$ of the context models (101 contributions) specify object properties below $40 \%$ of completeness. It indicates that a higher percentage of context models do not associate their classes with object properties to specify axioms such as "every instance of the Environment class must be related to instances of the Place class". However, the union between datatype and object properties indicates that a lower set (12 contributions, i.e. 9\%) of context models relates $100 \%$ of their classes with a datatype or object property. An extended report of the data used for this analysis can be found in online Annexes I and J at [17].

Table 3 condenses the most relevant information obtained throughout these two first research questions comprising the chronological overview and characteristics of the 138 reviewed proposals.

Table 3: Characteristics of the context models

\begin{tabular}{|c|c|c|c|c|c|c|c|c|c|}
\hline \multirow[b]{2}{*}{ Proposal } & \multirow[b]{2}{*}{ Formalism } & \multirow[b]{2}{*}{ Development method } & \multirow[b]{2}{*}{$\begin{array}{c}\text { Depth } \\
\text { level }\end{array}$} & \multirow[b]{2}{*}{$\begin{array}{c}\text { Clas- } \\
\text { ses }\end{array}$} & \multirow[b]{2}{*}{$\begin{array}{c}\text { Data- } \\
\text { type p. }\end{array}$} & \multirow[b]{2}{*}{$\begin{array}{c}\text { Object } \\
\text { p. }\end{array}$} & \multicolumn{3}{|c|}{ Definition completeness } \\
\hline & & & & & & & \begin{tabular}{c|c|} 
Context \\
information
\end{tabular} & \begin{tabular}{|l|} 
Datatype \\
property
\end{tabular} & \begin{tabular}{|c|}
$\begin{array}{c}\text { Object } \\
\text { property }\end{array}$ \\
\end{tabular} \\
\hline 1. Sliwa and Gleba [2015] & Ontology-based & 149 & 3 & 44 & 6 & 14 & $27 \%$ & $14 \%$ & $25 \%$ \\
\hline 2. Krömker and Wienken [2015] & Context taxonomy & 170 & 1 & 8 & 0 & 0 & $50 \%$ & $0 \%$ & $0 \%$ \\
\hline 3. Aly et al. [2015] & Ontology-based & none & 3 & 19 & 11 & 9 & $32 \%$ & $21 \%$ & $37 \%$ \\
\hline 4. Khemaja and Buendia [2015] & Ontology-based & 170 & 3 & 23 & 0 & 7 & $43 \%$ & $0 \%$ & $39 \%$ \\
\hline 5. Hoque et al. [2015] & Ontology-based & none & 4 & 51 & 5 & 8 & $0 \%$ & $6 \%$ & $12 \%$ \\
\hline 6. Hameurlaine et al. [2015] & Ontology-based & none & 3 & 22 & 9 & 11 & $45 \%$ & $18 \%$ & $32 \%$ \\
\hline 7. Khouja and Juiz [2015] & Ontology-based & none & 2 & 11 & 0 & 5 & $0 \%$ & $0 \%$ & $55 \%$ \\
\hline 8. Liang-Liang [2015] & Ontology-based & none & 1 & 5 & 0 & 4 & $100 \%$ & $0 \%$ & $100 \%$ \\
\hline 9. Saidani et al. [2015] & Ontology-based & 118,169 & 3 & 17 & 20 & 4 & $41 \%$ & $59 \%$ & $30 \%$ \\
\hline 10. Ghannem et al. [2015] & Ontology-based & None & 2 & 17 & 0 & 13 & $6 \%$ & $0 \%$ & $76 \%$ \\
\hline 11. Mok and Min [2015] & Ontology-based & None & 1 & 4 & 0 & 0 & $0 \%$ & $0 \%$ & $0 \%$ \\
\hline 12. Restrepo et al. [2014] & Ontology-based & None & 1 & 4 & 7 & 0 & $100 \%$ & $100 \%$ & $0 \%$ \\
\hline 13. Lee et al. [2014] & Context taxonomy & None & 2 & 14 & 0 & 0 & $100 \%$ & $0 \%$ & $0 \%$ \\
\hline 14. Xue et al. [2014] & Ontology-based & 139,140 & 4 & 37. & 1 & 11 & $51 \%$ & $3 \%$ & $30 \%$ \\
\hline 15. Wang and Tang [2014] & Ontology-based & None & 2 & 5 & 0 & 0 & $60 \%$ & $0 \%$ & $0 \%$ \\
\hline 16. Cabrera et al. [2014] & Ontology-based & 118,127 & 1 & 11 & 0 & 8 & $100 \%$ & $0 \%$ & $64 \%$ \\
\hline 17. Sohn et al. [2014] & Ontology-based & None & 3 & 13 & 8 & 0 & $8 \%$ & $62 \%$ & $0 \%$ \\
\hline 18. Yus et al. [2014] & Ontology-based & None & 3. & 9 & 2 & 4 & \begin{tabular}{l|l}
$0 \%$ \\
\end{tabular} & $11 \%$ & $44 \%$ \\
\hline 19. Choi et al. [2014] & Ontology-based & None & 4 & 59 & 0 & 0 & $26 \%$ & $0 \%$ & $0 \%$ \\
\hline 20. Sotsenko et al. [2014] & Context taxonomy & None & 2 & 17 & 0 & 0 & $24 \%$ & $0 \%$ & $0 \%$ \\
\hline 21. Li and Chen [2014] & Ontology-based & 141,142 & 2 & 8 & 0 & 0 & $88 \%$ & $0 \%$ & $0 \%$ \\
\hline 22. Chellouche et al. [2014] & Ontology-based & None & 2 & 36 & 0 & 17 & $11 \%$ & $0 \%$ & $61 \%$ \\
\hline 23. Zhang et al. [2014] & Ontology-based & None & 3 & 31 & 20 & 0 & $0 \%$ & $52 \%$ & $0 \%$ \\
\hline 24. Berri [2014] & Context taxonomy & None & 2 & 10 & 0 & 0 & $100 \%$ & $0 \%$ & $0 \%$ \\
\hline 25. Li et al. [2014] & Ontology-based & 118 & 2 & 9 & 0 & 0 & $33 \%$ & $0 \%$ & $0 \%$ \\
\hline 26. Katsumata [2014] & Ontology-based & None & 2 & 12 & 3 & 0 & $33 \%$ & $8 \%$ & $0 \%$ \\
\hline 27. Da et al. [2014] & Ontology-based & None & 1 & 4 & 0 & 4 & $50 \%$ & $0 \%$ & $100 \%$ \\
\hline 28. Benlamri and Zhang [2014] & Ontology-based & 149,172 & 2 & 26 & 14 & 31 & $65 \%$ & $31 \%$ & $81 \%$ \\
\hline 29. Miraoui [2014] & Ontology-based & none & 2 & 14 & 0 & 9 & $29 \%$ & $0 \%$ & $57 \%$ \\
\hline 30. Rahman et al. [2014] & Context taxonomy & none & 3 & 30 & 0 & 0 & $23 \%$ & $0 \%$ & $0 \%$ \\
\hline 31. Diallo et al. [2014] & Ontology-based & none & 4 & 47 & 3 & 2 & $13 \%$ & $0 \%$ & $68 \%$ \\
\hline 32. Maalej et al. [2014] & Ontology-based & none & 3 & 26 & 8 & 9 & $50 \%$ & $4 \%$ & $54 \%$ \\
\hline 33. Furno and Zimeo [2014] & Ontology-based & 155 & 3 & 18 & 0 & 5 & $22 \%$ & $0 \%$ & $28 \%$ \\
\hline 34. Novakovic and Huemer [2013] & Ontology-based & 143,144 & 4 & 16 & 0 & 0 & $13 \%$ & $0 \%$ & $0 \%$ \\
\hline 35. Ai et al. [2013] & Ontology-based & None & 3 & 18 & 0 & 8 & $61 \%$ & $0 \%$ & $50 \%$ \\
\hline 36. Lee and Kwon [2013] & Ontology-based & None & 1 & 3 & 0 & 0 & $100 \%$ & $0 \%$ & $0 \%$ \\
\hline 37. Villegas and Müller [2013] & Ontology-based & None & 4. & 25 & 24 & 37 & $100 \%$ & $28 \%$ & $72 \%$ \\
\hline 38. Kayes et al. [2013] & Ontology-based & None & 2 & 16 & 0 & 7 & $31 \%$ & $0 \%$ & $63 \%$ \\
\hline 39. Boudaa et al. [2013] & Ontology-based & None & 2 & 11 & 2 & 2 & \begin{tabular}{|l|l}
$55 \%$ & \\
\end{tabular} & $18 \%$ & $27 \%$ \\
\hline 40. Kim et al. [2013] & Ontology-based & None & 2 & 21 & 0 & 0 & \begin{tabular}{l|l}
$0 \%$ \\
\end{tabular} & $0 \%$ & $0 \%$ \\
\hline 41. Fissaa et al. [2013] & Ontology-based & None & 3 & 18 & 0 & 0 & $22 \%$ & \begin{tabular}{l|l}
$0 \%$ \\
\end{tabular} & $0 \%$ \\
\hline
\end{tabular}




\begin{tabular}{|c|c|c|c|c|c|c|c|c|c|}
\hline \multirow[b]{2}{*}{ Proposal } & \multirow[b]{2}{*}{ Formalism } & \multirow[b]{2}{*}{ Development method } & \multirow[b]{2}{*}{$\begin{array}{c}\text { Depth } \\
\text { level }\end{array}$} & \multirow[b]{2}{*}{$\begin{array}{c}\text { Clas- } \\
\text { ses }\end{array}$} & \multirow[b]{2}{*}{$\begin{array}{c}\text { Data- } \\
\text { type p. }\end{array}$} & \multirow[b]{2}{*}{$\begin{array}{c}\text { Object } \\
\text { p. }\end{array}$} & \multicolumn{3}{|c|}{ Definition completeness } \\
\hline & & & & & & & \begin{tabular}{c|c} 
Context \\
information
\end{tabular} & \begin{tabular}{|l|} 
Datatype \\
property
\end{tabular} & $\begin{array}{c}\text { Object } \\
\text { property }\end{array}$ \\
\hline 42. Zouhaier et al. [2013] & Ontology-based & None & 1 & 8 & 0 & 7 & $38 \%$ & $0 \%$ & $100 \%$ \\
\hline 43. Kabir et al. [2012] & Ontology-based & 145 & 1 & 4 & 0 & 4 & $100 \%$ & $0 \%$ & $100 \%$ \\
\hline 44. Rubio et al. [2012] & Ontology-based & $127,145,146,147$ & 3 & 9 & 20 & 13 & $67 \%$ & $89 \%$ & $33 \%$ \\
\hline 45. Paul.and Wachsmuth [2012] & Ontology-based & 148 & 1 & 8 & 0 & 0 & $0 \%$ & $0 \%$ & $0 \%$ \\
\hline 46. Hu et al. [2012] & Ontology-based & None & 1 & 5 & 0 & 5 & $0 \%$ & $0 \%$ & $100 \%$ \\
\hline 47. Copeland and Crespi [2012] & Context taxonomy & None & 1 & 6 & 0 & 0 & $100 \%$ & $0 \%$ & $0 \%$ \\
\hline 48. Wang et al. [2012] & Ontology-based & None & 3 & 15 & 0 & 0 & $0 \%$ & $0 \%$ & $0 \%$ \\
\hline 49. $\mathrm{Xu}$ and Dong [2012] & Ontology-based & none & 2 & 24 & 0 & 0 & $0 \%$ & $0 \%$ & $0 \%$ \\
\hline 50. Shen and Cheng [2011] & Ontology-based & 146 & 4 & 27 & 5 & 0 & $44 \%$ & $7 \%$ & $0 \%$ \\
\hline 51. Sheng et al. [2011] & Ontology-based & $118,145,149$ & 2 & 14 & 0 & 4 & $100 \%$ & $0 \%$ & $29 \%$ \\
\hline 52. Madkour and Maach [2011] & Ontology-based & 118 & 3 & 31 & 0 & 3 & $19 \%$ & $0 \%$ & $13 \%$ \\
\hline 53. Jang and Choi [2011] & Ontology-based & None & 4 & 39 & 15 & 20 & $0 \%$ & $13 \%$ & $46 \%$ \\
\hline 54. Gilman et al. [2011] & Ontology-based & None & 2 & 15 & 0 & 0 & $47 \%$ & $0 \%$ & $0 \%$ \\
\hline 55. Wusheng et al. [2011] & Ontology-based & None & 2 & 7 & 0 & 0 & $71 \%$ & $0 \%$ & $0 \%$ \\
\hline 56. $\operatorname{Li}[2011]$ & Ontology-based & None & 2 & 13 & 10 & 8 & $54 \%$ & $23 \%$ & $54 \%$ \\
\hline 57. Kim et al. [2011] & Ontology-based & None & 1 & 4 & 0 & 0 & $100 \%$ & $0 \%$ & $0 \%$ \\
\hline 58. Changboka et al. [2011] & Ontology-based & None & 4 & 16 & 7 & 1 & $19 \%$ & $13 \%$ & $13 \%$ \\
\hline 59. Hervás et al. [2010] & Ontology-based & 145 & 4 & 47 & 40 & 53 & $23 \%$ & $30 \%$ & $85 \%$ \\
\hline 60. Durán et al. [2010] & Ontology-based & 109 & 2 & 12 & 19 & 4 & $58 \%$ & $58 \%$ & $42 \%$ \\
\hline 61. Tong et al. [2010] & Ontology-based & None & 4 & 16 & 0 & 19 & $0 \%$ & $0 \%$ & $100 \%$ \\
\hline 62. Luo et al. [2010] & Ontology-based & None & 1 & 4 & 0 & 0 & $75 \%$ & $0 \%$ & $0 \%$ \\
\hline 63. Yi et al. [2010] & Ontology-based & None & 1 & 8 & 29 & 0 & $0 \%$ & $88 \%$ & $0 \%$ \\
\hline 64. Sun et al. [2010] & Ontology-based & None & 2 & 11 & 15 & 2 & $36 \%$ & $45 \%$ & $27 \%$ \\
\hline 65. Sigg et al. [2010] & Ontology-based & None & 3 & 67 & 0 & 0 & $0 \%$ & $0 \%$ & $0 \%$ \\
\hline 66. Park et al. [2010] & Ontology-based & None & 2 & 12 & 17 & 9 & $17 \%$ & $33 \%$ & $75 \%$ \\
\hline 67. Zainol and Nakata [2010] & Ontology-based & None & 3 & 13 & 38 & 0 & $31 \%$ & $62 \%$ & $0 \%$ \\
\hline 68. Chaker et al. [2010] & Context taxonomy & None & 1 & 3 & 0 & 0 & $100 \%$ & $0 \%$ & $0 \%$ \\
\hline 69. Saeedi et al.[2010] & Ontology-based & None & 2 & 11 & 0 & 0 & $18 \%$ & $0 \%$ & $0 \%$ \\
\hline 70. Bandara et al. [2009] & Ontology-based & 173 & 2 & 12 & 10 & 0 & $100 \%$ & $25 \%$ & $0 \%$ \\
\hline 71. Liu et al. [2009] & Ontology-based & 150 & 4 & 23 & 10 & 6 & $78 \%$ & $13 \%$ & $43 \%$ \\
\hline 72. Cadenas et al. [2009] & Ontology-based & $109,127,130$ & 1 & 10 & 0 & 11 & $100 \%$ & $0 \%$ & $100 \%$ \\
\hline 73. Hu and Li et al. [2009] & Ontology-based & None & 2 & 12 & 29 & 8 & $33 \%$ & \begin{tabular}{|l|l|}
$50 \%$ \\
\end{tabular} & $33 \%$ \\
\hline 74. Cioara et al. [2009] & Ontology-based & None & 2 & 5 & 0 & 0 & $60 \%$ & $0 \%$ & $0 \%$ \\
\hline 75. Gong et al. [2009] & Ontology-based & None & 4 & 27 & 0 & 5 & $26 \%$ & $0 \%$ & $33 \%$ \\
\hline 76. Cao et al. [2009] & Ontology-based & None & 2 & 9 & 14 & 4 & $0 \%$ & $56 \%$ & $44 \%$ \\
\hline 77. Najar et al. [2009] & Ontology-based & None & 3 & 19 & 0 & 1 & $47 \%$ & $0 \%$ & $11 \%$ \\
\hline 78. Lamsfus et al. [2009] & Ontology-based & 170 & 1 & 11 & 0 & 0 & $82 \%$ & $0 \%$ & $0 \%$ \\
\hline 79. Cao et al. [2008] & Ontology-based & 127 & 2 & 7 & 0 & 1 & $57 \%$ & $0 \%$ & $29 \%$ \\
\hline 80. Hur et al. [2008] & Ontology-based & 151 & 1 & 4 & 0 & 9 & $0 \%$ & $0 \%$ & $100 \%$ \\
\hline 81. Devaraju and Hoh [2008] & Ontology-based & $118,127,152,149$ & 4 & 39 & 0 & 1 & $77 \%$ & $0 \%$ & $5 \%$ \\
\hline 82. Coma et al. [2008] & Ontology-based & 146 & 3 & 14 & 2 & 2 & $86 \%$ & $14 \%$ & $14 \%$ \\
\hline 83. Strassner et al. [2008] & Ontology-based & 118 & 2 & 19 & 0 & 0 & $53 \%$ & $0 \%$ & $0 \%$ \\
\hline 84. Tan et al. [2008] & Context taxonomy & 153 & 4 & 46 & 0 & 0 & $13 \%$ & $0 \%$ & $0 \%$ \\
\hline 85. Sun et al. [2008] & Ontology-based & None & 4 & 32 & 5 & 8 & $0 \%$ & $9 \%$ & $22 \%$ \\
\hline 86. Ouyang et al. [2008] & Ontology-based & None & 2 & 18 & 6 & 5 & $61 \%$ & $11 \%$ & $28 \%$ \\
\hline 87. Kritsotakis et al. [2008] & Ontology-based & $149,167,168$ & 4 & 68 & 0 & 5 & $51 \%$ & $0 \%$ & $10 \%$ \\
\hline 88. Ko and $\operatorname{Sim}[2008]$ & Ontology-based & None & 3 & 19 & 7 & 5 & $21 \%$ & $11 \%$ & $21 \%$ \\
\hline 89. Pietschmann et al. [2008] & Ontology-based & $127,136,146,171$ & 4 & 67 & 40 & 56 & $100 \%$ & $15 \%$ & $63 \%$ \\
\hline 90. Chang et al. [2008] & Ontology-based & none & 2 & 7 & 0 & 4 & $100 \%$ & $0 \%$ & $29 \%$ \\
\hline 91. Ghadiri et al. [2007] & Ontology-based & \begin{tabular}{|l|l|}
132,154 \\
\end{tabular} & 4 & 39 & 0 & 0 & $28 \%$ & $0 \%$ & $0 \%$ \\
\hline 92. Park and Kwon [2007] & Ontology-based & $118,130,132,137,138$ & 1 & 15 & 0 & 0 & $80 \%$ & $0 \%$ & $0 \%$ \\
\hline 93. Erfianto et al. [2007] & Ontology-based & 107 & 3 & 13 & 3 & 0 & $31 \%$ & $15 \%$ & $0 \%$ \\
\hline 94. Qin et al. [2007] & Ontology-based & 146,155 & 3 & 17 & 17 & 13 & $24 \%$ & $29 \%$ & $41 \%$ \\
\hline 95. Lee and Meier [2007] & Ontology-based & None & 1 & 4 & 11 & 0 & $75 \%$ & $100 \%$ & $0 \%$ \\
\hline 96. Mei et al. [2007] & Context taxonomy & None & 1 & 5 & 0 & 0 & $100 \%$ & $0 \%$ & $0 \%$ \\
\hline 97. Paganelli et al. [2007] & Ontology-based & None & 2 & 11 & 0 & 6 & $55 \%$ & $0 \%$ & $55 \%$ \\
\hline 98. Zimmermann et al. [2007] & Context taxonomy & None & 2 & 12 & 0 & 0 & $100 \%$ & $0 \%$ & $0 \%$ \\
\hline 99. Hwang et al. [2007] & Ontology-based & None & 3 & 73 & 0 & 0 & $11 \%$ & $0 \%$ & $0 \%$ \\
\hline
\end{tabular}




\begin{tabular}{|c|c|c|c|c|c|c|c|c|c|}
\hline \multirow[b]{2}{*}{ Proposal } & \multirow[b]{2}{*}{ Formalism } & \multirow[b]{2}{*}{ Development method } & \multirow[b]{2}{*}{$\begin{array}{c}\text { Depth } \\
\text { level }\end{array}$} & \multirow[b]{2}{*}{$\begin{array}{c}\text { Clas- } \\
\text { ses }\end{array}$} & \multirow[b]{2}{*}{$\begin{array}{c}\text { Data- } \\
\text { type p. }\end{array}$} & \multirow[b]{2}{*}{$\begin{array}{c}\text { Object } \\
\text { p. }\end{array}$} & \multicolumn{3}{|c|}{ Definition completeness } \\
\hline & & & & & & & \begin{tabular}{c|c|} 
Context \\
information
\end{tabular} & \begin{tabular}{|l|} 
Datatype \\
property
\end{tabular} & $\begin{array}{c}\begin{array}{c}\text { Object } \\
\text { property }\end{array} \\
\end{array}$ \\
\hline 100. Choi and Yoon [2007] & Context taxonomy & None & 3 & 17 & 0 & 0 & $82 \%$ & $0 \%$ & $0 \%$ \\
\hline 101. Hwang et al. [2007] & Ontology-based & None & 1 & 6 & 0 & 7 & $17 \%$ & $0 \%$ & $100 \%$ \\
\hline 102. Jrad et al. [2007] & Ontology-based & 165,166 & 2 & 14 & 0 & 0 & $93 \%$ & $0 \%$ & $0 \%$ \\
\hline 103. Pessoa et al. [2007] & Ontology-based & None & 3 & 16 & 2 & 6 & $0 \%$ & $6 \%$ & $31 \%$ \\
\hline 104. Hamdeh and $\mathrm{Ma}$ [2007] & Ontology-based & 118 & 4 & 49 & 2 & 5 & $6 \%$ & $4 \%$ & $12 \%$ \\
\hline 105. Strimpakou et al. [2006] & Ontology-based & 129 & 2 & 18 & 0 & 32 & $67 \%$ & $0 \%$ & $78 \%$ \\
\hline 106. Sheng et al. [2006] & Ontology-based & 120 & 2 & 9 & 7 & 5 & $100 \%$ & $11 \%$ & $67 \%$ \\
\hline 107. Kim and Choi [2006] & Ontology-based & None & 4 & 27 & 15 & 15 & $0 \%$ & $22 \%$ & $37 \%$ \\
\hline 108. Wang et al. [2006] & Ontology-based & 145 & 1 & 7 & 25 & 0 & $100 \%$ & $57 \%$ & $0 \%$ \\
\hline 109. Heckmann [2006] & Ontology-based & 152,156 & 3 & 39 & 0 & 0 & $0 \%$ & $0 \%$ & $0 \%$ \\
\hline 110. Yang et al. [2006] & Ontology-based & None & 4 & 49 & 0 & 0 & $29 \%$ & $0 \%$ & $0 \%$ \\
\hline 111. Kranenburg et al. [2006] & Context taxonomy & None & 1 & 6 & 0 & 0 & $0 \%$ & $0 \%$ & $0 \%$ \\
\hline 112. Chaari et al. [2006] & Ontology-based & None & 1 & 7 & 0 & 0 & $0 \%$ & $0 \%$ & $0 \%$ \\
\hline 113. Ou et al. [2006] & Ontology-based & & 3 & 42 & 0 & 0 & $7 \%$ & $0 \%$ & $0 \%$ \\
\hline 114. Almeida et al. [2006] & Ontology-based & None & 2 & 11 & 0 & 10 & $64 \%$ & $0 \%$ & $100 \%$ \\
\hline 115. Hu and Moore [2006] & Ontology-based & None & 2 & 11 & 0 & 0 & $100 \%$ & $0 \%$ & $0 \%$ \\
\hline 116. Amundsen and Eliass. [2006] & Context taxonomy & None & 2 & 10 & 17 & 0 & $100 \%$ & $70 \%$ & $0 \%$ \\
\hline 117. Hong et al. [2006] & Ontology-based & None & 3 & 10 & 0 & 0 & $0 \%$ & $0 \%$ & $0 \%$ \\
\hline 118. Gu et al. [2005] & Ontology-based & None & 2 & 13 & 0 & 6 & $0 \%$ & $0 \%$ & $31 \%$ \\
\hline 119. Kalyan et al. [2005] & Ontology-based & 157 & 3 & 20 & 0 & 0 & $40 \%$ & $0 \%$ & $0 \%$ \\
\hline 120. Bu et al. [2005] & Ontology-based & None & 4 & 26 & 1 & 6 & $0 \%$ & $4 \%$ & $27 \%$ \\
\hline 121. Bradley and Dunlop [2005] & Context taxonomy & $158,159,160$ & 2 & 16 & 0 & 0 & $75 \%$ & $0 \%$ & $0 \%$ \\
\hline 122. Neto and Pimentel [2005] & Ontology-based & $145,146,147,149,152$ & 3 & 26 & 4 & 1 & $85 \%$ & $8 \%$ & $8 \%$ \\
\hline 123. Go and Sohn [2005] & Ontology-based & None & 1 & 5 & 0 & 0 & $100 \%$ & $0 \%$ & $0 \%$ \\
\hline 124. Zacarias et al. [2005] & Context taxonomy & None & 1 & 4 & 0 & 0 & $100 \%$ & $0 \%$ & $0 \%$ \\
\hline 125. Khedr and Karmouch [2005] & Ontology-based & None & 4 & 30 & 18 & 15 & $23 \%$ & $20 \%$ & $30 \%$ \\
\hline 126. Ko et al. [2005] & Ontology-based & \begin{tabular}{c|} 
None \\
\end{tabular} & 3 & 41 & 20 & 10 & $32 \%$ & $4 \%$ & $24 \%$ \\
\hline 127. Chen et al. [2004] & Ontology-based & $145,146,147,161,162$ & 2 & 20 & 26 & 24 & $100 \%$ & $35 \%$ & $75 \%$ \\
\hline 128. Kaenampor. and Neill [2004] & Ontology-based & \begin{tabular}{|c|}
163 \\
\end{tabular} & 2 & 22 & 0 & 0 & $73 \%$ & $0 \%$ & $0 \%$ \\
\hline 129. Xynogalas et al. [2004] & Context taxonomy & None & 2 & 28 & 0 & 0 & $100 \%$ & $0 \%$ & $0 \%$ \\
\hline 130. Preuveneers et al. [2004] & Ontology-based & $155,158,159$ & 4 & 38 & 15 & 24 & $45 \%$ & $16 \%$ & $55 \%$ \\
\hline 131. Ngo et al. [2004] & Ontology-based & $141,146,164$ & 4 & 97 & 5 & 19 & $30 \%$ & $1 \%$ & $25 \%$ \\
\hline 132. Hofer et al. [2003] & Context taxonomy & None & 1 & 5 & 0 & 0 & $100 \%$ & $0 \%$ & $0 \%$ \\
\hline 133. Tarasewich [2003] & Context taxonomy & 158 & 3 & 54 & 7 & 0 & $78 \%$ & $2 \%$ & $0 \%$ \\
\hline 134. Prekop et al. [2003] & Ontology-based & None & 2 & 7 & 0 & 0 & $43 \%$ & $0 \%$ & $29 \%$ \\
\hline 135. Korpipaa et al. [2003] & Ontology-based & None & 2 & 12 & 0 & 0 & $0 \%$ & $0 \%$ & $0 \%$ \\
\hline 136. Chen et al. [2003] & Ontology-based & None & 3 & 41 & 12 & 13 & $59 \%$ & $7 \%$ & $22 \%$ \\
\hline 137. Henricksen et al. [2002] & Ontology-based & None & 1 & 3 & 6 & 14 & $100 \%$ & $100 \%$ & $100 \%$ \\
\hline 138. Held et al. [2002] & Ontology-based & \begin{tabular}{|c|} 
None \\
\end{tabular} & 2 & 5 & 0 & 0 & $0 \%$ & $0 \%$ & $0 \%$ \\
\hline \multicolumn{10}{|c|}{$\begin{array}{l}\text { Identifiers: 139. SSN (Barnaghi et al. 2011); 140. DOLCE (Gangemi et al. 2003); 141. FIPA 2000; 142. BFO (Born et al. 2008); 143. FAO (Caracciolo et } \\
\text { al. 2008); 144. ISIC 2009; 145. FOAF (Brickley and Miller 2003); 146. OWL-Time/DAML-Time (Pan and Hobbs 2004); 147. OpenCyc (Lenat and Guha } \\
\text { 1990); 148. Dijk 1997; 149. CCPP (Klyne et al. 2004); 150. DogOnt (Bonino and Corno 2008); 151. Guarino 1998; 152. SUMO (Pease and Niles 2002); } \\
\text { 153. From } 78 \text { papers that were not referenced; 154. Maslow 1970; 155. OWL-S 2003; 156. Kobsa 2001; 157. Uschold et al. 1998; 158. Schmidt et al. } \\
\text { 1999; 159. Schilit et al. 1994; 160. Chen and Kotz 2000; 161. Randell et al. 1992; 162. MoGATU (Perich 2004); 163. Engeström et al. 1999; 164. NASA } \\
\text { Jet Propulsion Lab space ontology (http://sweet.jpl.nasa.gov/ontology/); 165. OntobUM (Razmerita et al. 2003); 166. Gavrilova et al. 2006; 167. } \\
\text { OntoNav's (Tsetsos et al. 2006); 168. Indoor Navigation Ontology (Kikiras et al. 2006); 169. Wang and Jiang 2012; 170. Dey et al. 2000; 171. PROTON } \\
\text { (http://proton.semanticweb.org/); 172. IEEELOM standard (http://Itsc.ieee.org/wg12/); 173. Rosemann et al. 2008 }\end{array}$} \\
\hline
\end{tabular}

\subsection{RQ1.3. Which classes of context information and entities are the most addressed in ontology- based context models?}

Throughout this question, we intended to analyze the most addressed context information classes and entities of the reviewed ontology-based context models. To address it, we grouped classes of context information and entities into hierarchies and synonyms that allowed us to compact the total of 2.756 terms coming from the 138 selected context models into 250 representative terms. This process involved the analysis of context term definitions provided in the proposals. From this analysis, we identified three 
representative structures as a pattern presented in the reviewed context models (see Figure 10). These structures are described as follows:

(A) Entity-dependent. Entities in a context model precede context information classes, i.e. each entity specified is characterized by different types of context information classes (e.g. User is an entity that can be characterized by State, Profile, Environment, etc.). Therefore, though the definition of an entity is intended to be generic, definitions of context information classes depend on the entity class from which they are related. A total of $44(31.88 \%)$ of the contributions follow this structure.

(B) Entity-independent. Entities and context information classes in a context model are also clearly separated, but to link them, relations such as aggregation or composition are necessary (e.g. entities such as Person, Organization and Service are related to context information classes such as Profile, Activity and Task). In this case, definitions of context information and entities classes are more generic. Only $16(11.59 \%)$ of the selected proposals follow this structure.

(C) Context-sensitive. Context models do not clearly separate entities from context information classes or vice versa. Therefore, definitions of classes of context information and entities can be as follows: the term used to define an entity also describes different types of context information classes (e.g. the entity Person refers to all humans and describes their Profiles, Status, Social relationships, etc.); terms used to specify entities are used to describe a specific context information class (e.g. the entity Person describes the Profile of a user); a term of a context information class can be used to characterize different entities (e.g. Profile refers to the profile of a Device, Person and Activity). This is the most adopted pattern with $78(56.52 \%)$ of the selected proposals following this structure.
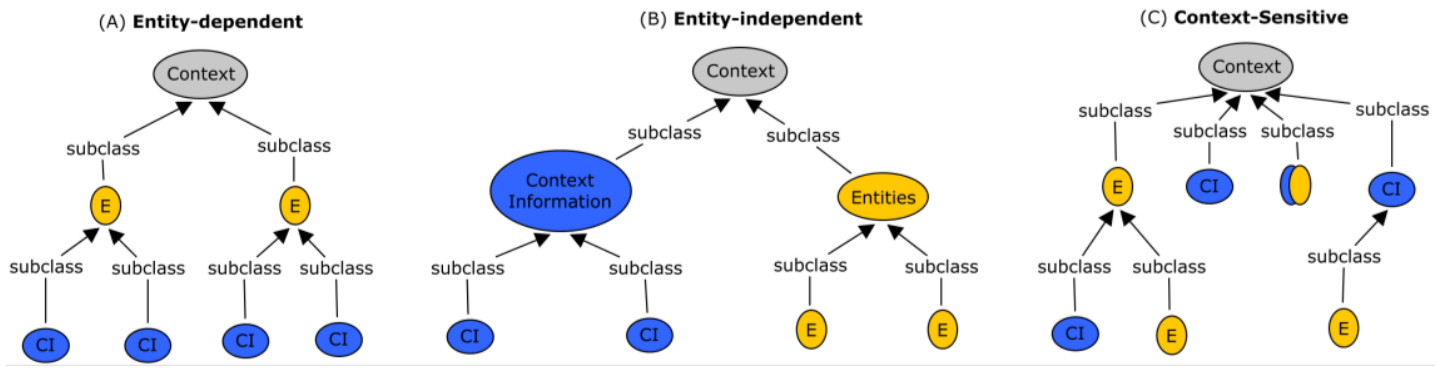

Figure 10: Structure pattern to specify and describe entities and context information classes

The findings previously identified were useful to establish the following criteria for grouping synonyms and hierarchize classes of context information and entities in order to consolidate the 2.756 existing terms:

- Terms whose definition describes a generic entity class (e.g. "a Service is a resource to deliver value") or generic context information class (e.g. "Activity describes the activities of agents") are positioned in the same level, preceding specific classes of context information or entities (e.g. Activity precedes UserActivity).

- Terms defining solely entities (e.g. "a Person refers to all users of a system") are grouped as synonyms with terms defining entities that also describe context (e.g. "Person describes the context information class of a user") if and when they refer to a generic description and an exact or similar meaning.

- Terms whose definition describes an entity class that refers to a specific context information class (e.g., the term "Person" describes the profile of users) are grouped as synonyms with the term describing this specific context information class (e.g. "Profile describes the profile of a Person") if and when the entities characterized belong to the same type (e.g. Person and User belong to humans).

- Whether some definition was not provided, we consider the parent and children classes specified for each term, i.e. if the term "Profile" is not defined, but the parent class is a Person or its sub-classes are context information classes of a Person, clearly it refers to a specific context of a Person. 
Table 4 shows the most representative synonyms and hierarchies as a result of applying the previous criteria in the selected context models, i.e. terms with more matches found in these models (see Table 5). In both tables we attempt to separate entities from context information classes in order to avoid blending them, issue presented in different proposals that might cause reusability problems. The complete analysis and an extended report of synonyms and hierarchies can be found in the online Annex K at [17].

Table 4: Table of synonyms and hierarchies

\begin{tabular}{|c|c|c|c|}
\hline Class & Second level & Third level & Synonyms \\
\hline \multicolumn{4}{|r|}{ Entities classes } \\
\hline \multirow[t]{4}{*}{ Agent } & & & Actors, SocialEntity, User, UserDomain, Participant, Entity \\
\hline & Person & & $\begin{array}{l}\text { People, OtherPeople, Individual, Personal, Human, HumanEntity, } \\
\text { Visitor }\end{array}$ \\
\hline & & User & $\begin{array}{l}\text { Who, User "fingerprints", UserViewInformation, Participants, Users, } \\
\text { IntrinsicContext, UserDriver, UserInformation, UserEntity }\end{array}$ \\
\hline & Organization & & Org, OrganizationRelationship, Organizational \\
\hline \multirow[t]{5}{*}{ Resource } & & & - \\
\hline & $\begin{array}{l}\text { Computational } \\
\text { Entity }\end{array}$ & & $\begin{array}{l}\text { CompEntity, Computational, Computing, Computing\&Connectivity, } \\
\text { SystemViewInformation, ComputingDomain, Platform, } \\
\text { ComputingEnvironment }\end{array}$ \\
\hline & & Device & $\begin{array}{l}\text { Entity, ComputingEntity, Resources, ComputingDevices, Platform, } \\
\text { Devices, how, Computation, ServiceObject, ComputationalDevice, } \\
\text { TecnologicContext, DeviceContext }\end{array}$ \\
\hline & & Service & ServiceViewInformation, Services \\
\hline & & Network & Connectivity, NetworkEntity, NetworkConnectivity \\
\hline \multicolumn{4}{|c|}{ Context information classes } \\
\hline \multirow[t]{4}{*}{ Activity } & & & $\begin{array}{l}\text { Activities, what, UserActivity, ActivityProcess, SocialActivity, } \\
\text { CommunityActivities, ActivityEntity }\end{array}$ \\
\hline & Task & & Tasks, CommunityTasks, AssociatedTasks \\
\hline & Event & & Events, ActivityEvent \\
\hline & Agenda & & ScheduledEvent, Calendar, Timetable, Scheduled, ScheduledActivity \\
\hline Time & & & $\begin{array}{l}\text { Time-related, When, Time, when, Temporal, TimeExpression, } \\
\text { History, Time-based, TemporalInformation, TemporalInfo, } \\
\text { TemporalThing, TimeContext }\end{array}$ \\
\hline \multirow[t]{4}{*}{ Environment } & & & Environmental, ImmediateEnvironment, Extrinsic \\
\hline & $\begin{array}{l}\text { Physical } \\
\text { Environment }\end{array}$ & & $\begin{array}{l}\text { EnvironmentalConditions, EnvironmentalSensorData, Environment, } \\
\text { Climate, Physical, PhysicalAspects, Weather, Physics, } \\
\text { PhysicalConditions, ClimateCondition, PhysicalContext, } \\
\text { UserEnvironmentDescription, EnvironmentalContext, PhysicalEntity }\end{array}$ \\
\hline & $\begin{array}{l}\text { Social } \\
\text { Environment }\end{array}$ & & User’sSocialEnvironment, Social, SocialContext, HumanFactors \\
\hline & & $\begin{array}{l}\text { Relation- } \\
\text { ships }\end{array}$ & SocialRelations, SocialRelationships, SocialRelationshipData \\
\hline \multirow[t]{3}{*}{ Location } & & & $\begin{array}{l}\text { Co-location, Place, Where, LocationSensor, Space, Locality, } \\
\text { LocationFeature, GeographicLocations, PhysicalLocation, } \\
\text { Spatial_Context, LocationEntity, SpationTemporalContext }\end{array}$ \\
\hline & Outdoor & & OutdoorSpace, OutsideHome, OutdoorPlace \\
\hline & Indoor & & IndoorSpace, In-doorSpace, IndoorPlace \\
\hline \multirow[t]{3}{*}{ Profile } & & & Profiles, DefaultProfiles, ProfileInfo \\
\hline & UserProfile & & $\begin{array}{l}\text { Person, PersonProfile, PersonalInformation, ProfileInformation, } \\
\text { UserPersonalDescription, UserCharacteristics, UserObject, } \\
\text { PersonalProfile }\end{array}$ \\
\hline & Preferences & & $\begin{array}{l}\text { UserPersonalPreferencesAndInterests, UserPreferences, } \\
\text { UserPreferenceDescription }\end{array}$ \\
\hline
\end{tabular}




\begin{tabular}{lll}
\hline Role & & PersonRole, DivisionOfLabour, AgentRole, SocialRole \\
\hline $\begin{array}{l}\text { StatesAnd } \\
\text { Status }\end{array}$ & & $\begin{array}{l}\text { UserCurrentState, State, CurrentState, Status, StatusInfo, } \\
\text { CurrentStateDescription, CurrentStatus }\end{array}$ \\
\hline & $\begin{array}{l}\text { Physiological } \\
\text { States }\end{array}$ & Physiological, Physiologic, BioCondition, BiomedicalSensorData \\
& Emotion & $\begin{array}{l}\text { UserEmotionalState, EmotionalState, Emotional, } \\
\text { Emotional/Mental, EmotionalSituation, Mental state }\end{array}$ \\
\hline
\end{tabular}

As can be seen in Table 4, the context information class with higher number of synonyms is Physical Environment that considers 14 terms with exact or similar meaning, followed by Location, Time and Device with 12 terms each, User with 9 terms, and Computational entity and UserProfile with 8 terms each. Contrary to them, the class Resource is used consistently in all the selected context models, i.e. the term used to define it is always the same. We also identified three features regarding terms used to specify classes of context information and entities, described as follows: some terms maintain a regular variation of the word (e.g. "Physiological states" and most of its synonyms present a slight variation among them); some terms are highly generic or very specific (e.g. synonyms of "Physical environment" such as "Environment" and "User environment description"); finally, although the terms maintain the same meaning some of them seem to be very distinct (e.g. "Environment" and "Extrinsic").

These inconsistencies affect the reuse of context models especially if it is preferred to create new terms instead of verifying which models provide the vocabulary semantically coherent with the terms identified. In this sense, the integration of more than one model should be documented for every term whose definition is going to be used [23]. It is worth to remark that we identified terms representing entities; however, most of these terms were also used to describe context information classes in several proposals as noted above.

Regarding matches and hierarchy levels of classes of context information and entities depicted in Table 5, we found also inconsistencies related to how this information is hierarchized among the analysed context models. As can be seen in the table, none of the terms remains constant into the same hierarchy level; they appear at different levels at least once. Terms with slight variability into the specified hierarchy levels and that seem to be more consolidated in a single one, i.e. terms that in a higher percentage remain constant into the same hierarchy level, are User, Organization, Tasks, Agenda, Environment, Outdoor, Indoor and UserProfile. It is worth noting that we have hierarchized classes of context information and entities based on the consolidated hierarchy levels of the terms and considering generic definitions that can include a higher number of terms. In this sense, although the term User provides 34 matches at first level hierarchy, Person and Agent are more generic according to their definitions. A special case was identified with the term Profile: only 6 models (4.3\%) provides a generic definition of this context information class, most of proposals refer to a User profile. However, we consider the term Profile in the hierarchy for grouping more terms such as Device profile, Activity profile, etc.

Table 5: Table of matches and hierarchies

\begin{tabular}{|c|c|c|c|c|c|c|c|}
\hline \multirow{2}{*}{ Class } & \multirow{2}{*}{ Second level } & \multirow{2}{*}{ Third level } & \multirow{2}{*}{ Matches } & \multicolumn{4}{|c|}{ Hierarchy levels } \\
\hline & & & & 1st & 2nd & 3rd & 4th \\
\hline \multicolumn{8}{|c|}{ Entities classes } \\
\hline \multirow[t]{4}{*}{ Agent } & & & 22 & 14 & 5 & 2 & 1 \\
\hline & Person & & 36 & 15 & 14 & 5 & 2 \\
\hline & & User & 41 & 34 & 6 & 1 & 0 \\
\hline & Organization & & 9 & 1 & 7 & 1 & 0 \\
\hline \multirow[t]{4}{*}{ Resource } & & & 12 & 5 & 5 & 2 & 0 \\
\hline & ComputationalEntity & & 12 & 5 & 5 & 2 & 0 \\
\hline & & Device & 49 & 22 & 19 & 6 & 2 \\
\hline & & Service & 28 & 14 & 9 & 4 & 1 \\
\hline
\end{tabular}




\begin{tabular}{|c|c|c|c|c|c|c|c|}
\hline & & Network & 30 & 14 & 9 & 5 & 2 \\
\hline \multicolumn{8}{|c|}{ Context information classes } \\
\hline \multirow[t]{4}{*}{ Activity } & & & 68 & 34 & 27 & 5 & 2 \\
\hline & Task & & 19 & 5 & 12 & 0 & 2 \\
\hline & Event & & 14 & 6 & 6 & 2 & 0 \\
\hline & Agenda & & 19 & 3 & 12 & 4 & 0 \\
\hline Time & & & 71 & 34 & 31 & 5 & 1 \\
\hline \multirow[t]{4}{*}{ Environment } & & & 24 & 18 & 5 & 1 & 0 \\
\hline & PhysicalEnvironment & & 32 & 14 & 15 & 3 & 0 \\
\hline & SocialEnvironment & & 15 & 7 & 6 & 2 & 0 \\
\hline & & Relationships & 11 & 3 & 5 & 2 & 1 \\
\hline \multirow[t]{3}{*}{ Location } & & & 92 & 40 & 40 & 11 & 1 \\
\hline & Outdoor & & 14 & 0 & 5 & 8 & 1 \\
\hline & Indoor & & 14 & 0 & 5 & 8 & 1 \\
\hline \multirow[t]{3}{*}{ Profile } & & & 6 & 5 & 1 & 0 & 0 \\
\hline & UserProfile & & 43 & 12 & 22 & 7 & 2 \\
\hline & Preferences & & 25 & 8 & 9 & 7 & 1 \\
\hline Role & & & 25 & 6 & 11 & 6 & 2 \\
\hline \multirow[t]{3}{*}{ StatesAndStatus } & & & 12 & 4 & 4 & 4 & 0 \\
\hline & PhysiologicalStates & & 10 & 1 & 5 & 4 & 0 \\
\hline & Emotion & & 12 & 0 & 7 & 5 & 0 \\
\hline
\end{tabular}

Once classes of context information and entities were grouped and hierarchized, we analysed which is the most addressed context information class of the selected context models. To do so, we used the first level classes previously established as a reference for the comparison. To evaluate the coverage of these classes on each context model, we defined the following criteria:

- Explicitly defined without divisions $(\checkmark)$. Describes whether a class is explicitly defined in the context model.

- Explicitly defined with divisions $(\checkmark *)$. Describes whether a class is explicitly defined in the context model with further subclasses.

- Partially defined "reduced" (P). Describes whether a class is not explicitly defined, but the model has a class or subclass which can be classified into this class.

- Partially defined "extended" $\left(\mathrm{P}^{*}\right)$. Describes whether a class is not explicitly defined, but the context model has different classes or subclasses which can be classified into this class.

- Not defined (X). Describes whether a class is not explicitly or partially defined.

Table 6 reports the results of applying the previous criteria on the selected context models. At the end of this table, the percentages obtained for each criteria are specified. As shown, none of the classes are covered $100 \%$ in the context models. Regarding context information classes, the most addressed class is the Location class representing $65 \%$ of the classes explicitly defined with or without further hierarchies, followed by Time, Activity and Environment with 50\%, 48\%, and 31\% respectively.

The explicit definition of the remaining context information classes in the context models decreases considerably with respect to those discussed above. None of them is explicitly defined by more than $28 \%$ of the proposals: Profile is defined in $26 \%$ of the proposals followed by Role with 16\%, and States\&Status with $9 \%$. However, they are covered in context models by defining some of their sub-characteristics, i.e. partially defined. The results show that proposals partially cover Profile, Role and States\&Status classes in $64 \%, 40 \%$ and $36 \%$ respectively. The percentage of classes that were not defined (neither explicitly nor partially) in the analysed context models is slightly high and significant, especially in context information classes such as States\&Status with 55\%, followed by Time and Role with $46 \%$ and $43 \%$ respectively. 
Regarding classes of entities, most of the proposals do not provide neither an explicit definition of the Agent class that represents entities such as Person, Organism, etc., nor Resource that represent Services, Devices, etc. In fact, only $19 \%$ and $15 \%$ of the contributions explicitly define the Agent and Resource classes respectively with or without further hierarchies. Other $61 \%$ and $69 \%$ of the proposals, respectively, do partially define them, i.e., define some of their sub-characteristics. Finally, $37 \%$ of the proposals do not specify any entity and therefore, they prefer to characterize only context information that can be applied to different entities beyond focused on specific ones. Note that these classes (context information and entities) were previously selected by means of matches in the proposals (see Table 5), i.e. several classes are completely absent in a considerable set of context models, especially those that are specific in a domain.

Table 6: Context information classes and entities coverage

\begin{tabular}{|c|c|c|c|c|c|c|c|c|c|}
\hline Proposals & Agent & Resource & Activity & Time & $\begin{array}{c}\text { Enviro } \\
\text { nment }\end{array}$ & Location & Profile & Role & $\begin{array}{c}\text { States \& } \\
\text { Status }\end{array}$ \\
\hline Henricksen et al. [M1] & $\mathrm{P}$ & $\mathrm{P}^{*}$ & $\mathrm{X}$ & $\mathrm{X}$ & $\mathrm{X}$ & $\mathrm{X}$ & $\mathrm{X}$ & $\mathrm{X}$ & $\mathrm{X}$ \\
\hline Held et al. [M2] & $\mathrm{P}$ & $\mathrm{P}^{*}$ & $\mathrm{X}$ & $\mathrm{X}$ & $\checkmark$ & $\mathrm{X}$ & $\checkmark$ & $\mathrm{X}$ & $\mathrm{X}$ \\
\hline Tarasewich [M3] & $\mathrm{P}$ & $\mathrm{P}$ & $\sqrt{ } *$ & $\sqrt{ } *$ & $\sqrt{ } *$ & $\checkmark$ & $\mathrm{P}^{*}$ & $\mathrm{X}$ & $\sqrt{ } *$ \\
\hline Ghannem et al. [M4] & $\mathrm{P}$ & $\mathrm{P}^{*}$ & $\mathrm{X}$ & $\mathrm{X}$ & $\checkmark$ & $\mathrm{P}^{*}$ & $\mathrm{X}$ & $\mathrm{X}$ & $\mathrm{X}$ \\
\hline Mok and Min [M5] & $\mathrm{X}$ & $\mathrm{X}$ & $\checkmark$ & $\checkmark$ & $\mathrm{X}$ & $\checkmark$ & $\mathrm{P}$ & $\mathrm{X}$ & $\mathrm{X}$ \\
\hline Chen et al. [M6] & $\checkmark$ & $\mathrm{P}^{*}$ & $\mathrm{P}^{*}$ & $\checkmark$ & $\mathrm{P}$ & $\checkmark$ & $\checkmark$ & $\mathrm{X}$ & $\mathrm{X}$ \\
\hline Cao et al. [M7] & $\mathrm{P}$ & $\mathrm{P}$ & $\checkmark$ & $\checkmark$ & $\mathrm{P}$ & $\checkmark$ & $\mathrm{P}^{*}$ & $\mathrm{P}$ & $\mathrm{X}$ \\
\hline Devaraju and Hoh [M8] & $\checkmark *$ & $\sqrt{ } *$ & $\checkmark$ & $\mathrm{X}$ & $\mathrm{P}$ & $\checkmark$ & $\mathrm{P}^{*}$ & $\mathrm{P}$ & $\mathrm{P}$ \\
\hline Pietschmann et al. [M9] & $\checkmark *$ & $\mathrm{P}^{*}$ & $\sqrt{ } *$ & $\sqrt{ } *$ & $X$ & $\checkmark *$ & $\checkmark$ & $\checkmark$ & $\checkmark$ \\
\hline Cadenas et al. [M10] & $\mathrm{P}$ & $\mathrm{P}^{*}$ & $\mathrm{X}$ & $\checkmark$ & $\checkmark$ & $\checkmark$ & $\mathrm{P}^{*}$ & $\checkmark$ & $\mathrm{P}$ \\
\hline Rubio et al. [M11] & $\mathrm{P}$ & $\mathrm{X}$ & $\mathrm{P}$ & $\checkmark$ & $\sqrt{ } *$ & $\checkmark *$ & $\mathrm{P}^{*}$ & $\mathrm{P}$ & $\mathrm{X}$ \\
\hline Cabrera et al. [M12] & $\mathrm{X}$ & $\checkmark$ & $\checkmark$ & $\checkmark$ & $\checkmark$ & $\checkmark$ & $\checkmark$ & $\checkmark$ & $\mathrm{X}$ \\
\hline Gu et al. [M13] & $\checkmark$ & $\mathrm{P}^{*}$ & $\checkmark *$ & $\mathrm{X}$ & $\mathrm{P}$ & $\sqrt{ } *$ & $\mathrm{P}^{*}$ & $\mathrm{X}$ & $\mathrm{X}$ \\
\hline Hofer et al. [M14] & $\mathrm{P}$ & $\mathrm{P}^{*}$ & $\mathrm{X}$ & $\checkmark$ & $X$ & $\checkmark$ & $\mathrm{P}^{*}$ & $\mathrm{X}$ & $\mathrm{X}$ \\
\hline Preuveneers et al. [M15] & $\mathrm{P}$ & $\checkmark *$ & $\checkmark$ & $\checkmark$ & $\checkmark *$ & $\sqrt{ } *$ & $\checkmark *$ & $\checkmark$ & $\mathrm{P}^{*}$ \\
\hline Heckmann [M16] & $\mathrm{P}$ & $\mathrm{P}$ & $\mathrm{X}$ & $\mathrm{X}$ & $\mathrm{P}$ & $\mathrm{P}$ & $\mathrm{P} *$ & $\checkmark$ & $\mathrm{P}^{*}$ \\
\hline Chen et al. [M17] & $\checkmark *$ & $\mathrm{P}^{*}$ & $\sqrt{ } *$ & $\mathrm{X}$ & $\mathrm{X}$ & $\sqrt{ } *$ & $\mathrm{P}^{*}$ & $\checkmark *$ & $\mathrm{X}$ \\
\hline Xynogalas et al. [M18] & $\mathrm{P}$ & $\mathrm{P}^{*}$ & $\mathrm{X}$ & $\checkmark$ & $\mathrm{P}$ & $\sqrt{ } *$ & $\checkmark$ & $\mathrm{X}$ & $\sqrt{ } *$ \\
\hline Bu et al. [M19] & $\sqrt{ } *$ & $\mathrm{P}^{*}$ & $\mathrm{X}$ & $\mathrm{X}$ & $\mathrm{X}$ & $\mathrm{X}$ & $\mathrm{P}^{*}$ & $\mathrm{X}$ & $\mathrm{X}$ \\
\hline Kim and Choi [M20] & $\checkmark *$ & $\mathrm{P}^{*}$ & $\checkmark$ & $\checkmark$ & $\mathrm{P}$ & $\sqrt{ } *$ & $\mathrm{P} *$ & $\mathrm{X}$ & $\mathrm{X}$ \\
\hline Neto and Pimentel [M21] & $\sqrt{ } *$ & $\mathrm{P}^{*}$ & $\sqrt{ } *$ & $\sqrt{ } *$ & $\mathrm{P}$ & $\sqrt{ } *$ & $\sqrt{ } *$ & $\checkmark$ & $\mathrm{X}$ \\
\hline Park and Kwon [M22] & $\mathrm{P}^{*}$ & $\mathrm{P}^{*}$ & $\checkmark$ & $\checkmark$ & $\checkmark$ & $\checkmark$ & $\mathrm{P}$ & $\checkmark$ & $\mathrm{X}$ \\
\hline Ngo et al. [M23] & $\checkmark *$ & $\mathrm{P}^{*}$ & $\sqrt{ } *$ & $\sqrt{ } *$ & $\sqrt{ } *$ & $\sqrt{ } *$ & $\sqrt{ } *$ & $\mathrm{X}$ & $\mathrm{X}$ \\
\hline Bradley and Dunlop [M24] & $\mathrm{P}^{*}$ & $\mathrm{P}^{*}$ & $\mathrm{P}^{*}$ & $\mathrm{P}$ & $\checkmark$ & $\mathrm{P}$ & $\mathrm{P}$ & $\mathrm{P}$ & $\mathrm{P}$ \\
\hline Kritsotakis et al. [M25] & $\mathrm{P}^{*}$ & $\mathrm{P}^{*}$ & $\mathrm{P}$ & $\mathrm{X}$ & $\mathrm{X}$ & $\sqrt{ } *$ & $\checkmark$ & $\mathrm{P}^{*}$ & $\mathrm{P}^{*}$ \\
\hline Sheng et al. [M26] & $\mathrm{P}^{*}$ & $\mathrm{P}^{*}$ & $\sqrt{ } *$ & $\mathrm{X}$ & $\mathrm{P}$ & $\checkmark *$ & $\checkmark$ & $\mathrm{X}$ & $\mathrm{X}$ \\
\hline Lee and Kwon [M27] & $\mathrm{X}$ & $\mathrm{P}$ & $\mathrm{X}$ & $\mathrm{X}$ & $\mathrm{P}^{*}$ & $\mathrm{X}$ & $\mathrm{X}$ & $\mathrm{X}$ & $\mathrm{P}$ \\
\hline Chaker et al. [M28] & $\mathrm{P}$ & $\mathrm{X}$ & $\mathrm{P}$ & $\mathrm{X}$ & $\checkmark$ & $\mathrm{X}$ & $\mathrm{P}^{*}$ & $\mathrm{P}$ & $\mathrm{P}$ \\
\hline Hervás et al. [M29] & $\mathrm{P} *$ & $\mathrm{P}^{*}$ & $\mathrm{P}^{*}$ & $\mathrm{X}$ & $\mathrm{P}^{*}$ & $\sqrt{ } *$ & $\checkmark$ & $\checkmark$ & $\checkmark$ \\
\hline Najar et al. [M30] & $\sqrt{ } *$ & $\checkmark *$ & $\mathrm{P} *$ & $\checkmark$ & $\mathrm{P}$ & $\checkmark$ & $\mathrm{P}^{*}$ & $\checkmark$ & $\mathrm{X}$ \\
\hline Prekop et al. [M31] & $\checkmark$ & $\checkmark *$ & $\checkmark *$ & $\mathrm{X}$ & $\mathrm{X}$ & $\mathrm{X}$ & $\mathrm{P}$ & $\mathrm{X}$ & $\mathrm{P}$ \\
\hline Khedr and Karmouch [M32] & $\sqrt{ } *$ & $\mathrm{P}^{*}$ & $\checkmark$ & $\checkmark$ & $\mathrm{P}$ & $\sqrt{ } *$ & $\checkmark$ & $\checkmark$ & $\mathrm{P}$ \\
\hline Gong et al. [M33] & $\mathrm{P} *$ & $\sqrt{ } *$ & $\mathrm{P}$ & $\mathrm{X}$ & $\mathrm{X}$ & $\mathrm{X}$ & $\mathrm{P}^{*}$ & $\mathrm{P}^{*}$ & $\mathrm{X}$ \\
\hline Villegas and Müller [M34] & $\checkmark *$ & $\mathrm{P}^{*}$ & $\sqrt{ } *$ & $\sqrt{ } *$ & $\mathrm{P}$ & $\sqrt{ } *$ & $\mathrm{P}^{*}$ & $\mathrm{X}$ & $\mathrm{P}$ \\
\hline Strassner et al. [M35] & $\sqrt{ } *$ & $\checkmark$ & $\checkmark *$ & $\sqrt{ } *$ & $\mathrm{P}$ & $\sqrt{ } *$ & $\mathrm{P}^{*}$ & $\mathrm{P}$ & $\mathrm{X}$ \\
\hline Zimmermann et al. [M36] & $\checkmark *$ & $\mathrm{P}^{*}$ & $\checkmark$ & $\checkmark$ & $\mathrm{P}$ & $\checkmark$ & $\mathrm{P}^{*}$ & $\mathrm{P}$ & $\mathrm{P}^{*}$ \\
\hline Shen and Cheng [M37] & $\checkmark$ & $\checkmark *$ & $\checkmark *$ & $\checkmark$ & $\checkmark$ & $\sqrt{ } *$ & $\mathrm{P}^{*}$ & $\mathrm{P}^{*}$ & $\mathrm{X}$ \\
\hline Kayes et al. [M38] & $\checkmark *$ & $\checkmark$ & $\mathrm{P}$ & $\checkmark$ & $\mathrm{P}$ & $\checkmark$ & $\checkmark$ & $\checkmark$ & $\checkmark$ \\
\hline Śliwa and Gleba [M39] & $\mathrm{P}$ & $\mathrm{P}^{*}$ & $\mathrm{P}^{*}$ & $\mathrm{X}$ & $\mathrm{X}$ & $\mathrm{X}$ & $\checkmark$ & $\mathrm{P}$ & $\mathrm{X}$ \\
\hline Krömker and Wienken [M40] & $\mathrm{P}$ & $\mathrm{X}$ & $\mathrm{X}$ & $\mathrm{X}$ & $\mathrm{P}^{*}$ & $\checkmark$ & $\mathrm{P}$ & $\mathrm{P}$ & $\mathrm{X}$ \\
\hline Aly et al. [M41] & $\mathrm{P}$ & $\mathrm{P}^{*}$ & $\mathrm{X}$ & $\mathrm{X}$ & $\mathrm{X}$ & $\checkmark *$ & $\mathrm{X}$ & $\mathrm{X}$ & $\mathrm{X}$ \\
\hline Khemaja and Buendía [M42] & $\mathrm{P}^{*}$ & $\mathrm{P}$ & $\sqrt{ } *$ & $\checkmark$ & $\mathrm{P} *$ & $\sqrt{ } *$ & $\mathrm{P}$ & $\mathrm{P}^{*}$ & $\mathrm{X}$ \\
\hline Hoque et al. [M43] & $\mathrm{P}^{*}$ & $\mathrm{P}^{*}$ & $\sqrt{ } *$ & $\sqrt{ } *$ & $\mathrm{P}^{*}$ & $\sqrt{ } *$ & $\mathrm{X}$ & $\mathrm{P}^{*}$ & $\mathrm{X}$ \\
\hline Hameurlaine et al. [M44] & $\mathrm{X}$ & $\mathrm{P}^{*}$ & $\mathrm{X}$ & $\checkmark$ & $\mathrm{P} *$ & $\checkmark$ & $\sqrt{ } *$ & $\mathrm{P}^{*}$ & $\mathrm{X}$ \\
\hline Khouja and Juiz [M45] & $\mathrm{P}$ & $\checkmark$ & $\mathrm{X}$ & $\mathrm{X}$ & $\mathrm{X}$ & $X$ & $\mathrm{X}$ & $\mathrm{P}^{*}$ & $\mathrm{X}$ \\
\hline
\end{tabular}




\begin{tabular}{|c|c|c|c|c|c|c|c|c|c|}
\hline Proposals & Agent & Resource & Activity & Time & $\begin{array}{c}\text { Enviro } \\
\text { nment }\end{array}$ & Location & Profile & Role & $\begin{array}{c}\text { States \& } \\
\text { Status }\end{array}$ \\
\hline Liang-Liang [M46] & $\mathrm{X}$ & $\checkmark$ & $\mathrm{X}$ & $\mathrm{X}$ & $\mathrm{X}$ & $\mathrm{X}$ & $\mathrm{P}^{*}$ & $\mathrm{X}$ & $\mathrm{X}$ \\
\hline Saidani et al. [M47] & $\mathrm{P}$ & $\checkmark *$ & $\mathrm{P}$ & $\checkmark$ & $\checkmark *$ & $\checkmark$ & $\mathrm{P}^{*}$ & $\checkmark$ & $\mathrm{X}$ \\
\hline Restrepo et al. [M48] & $\mathrm{X}$ & $\mathrm{P}$ & $\mathrm{P}$ & $\mathrm{P}$ & $\mathrm{P}^{*}$ & $\mathrm{P}$ & $\mathrm{P}$ & $\mathrm{P}$ & $\mathrm{P}$ \\
\hline Lee et al. [M49] & $\mathrm{X}$ & $\mathrm{X}$ & $\mathrm{P}^{*}$ & $\checkmark$ & P* & P* & $\mathrm{X}$ & $\mathrm{X}$ & $\mathrm{P}$ \\
\hline Xue et al. [M50] & $\mathrm{X}$ & $\mathrm{P}^{*}$ & $\mathrm{X}$ & $\mathrm{X}$ & $\mathrm{P}^{*}$ & $\mathrm{P}^{*}$ & $\mathrm{P}$ & $\mathrm{X}$ & $\checkmark$ \\
\hline Wang and Tang [M51] & $\checkmark$ & $\checkmark *$ & $\mathrm{X}$ & $\mathrm{X}$ & $\mathrm{X}$ & $\mathrm{X}$ & $\mathrm{P}^{*}$ & $\mathrm{X}$ & $\mathrm{P}$ \\
\hline Sohn et al. [M52] & $\mathrm{P}$ & $\mathrm{X}$ & $\mathrm{X}$ & $\checkmark$ & $\sqrt{ } *$ & $\mathrm{P}$ & $\checkmark$ & $\mathrm{X}$ & $\mathrm{X}$ \\
\hline Yus et al. [M53] & $\mathrm{P}$ & $\mathrm{X}$ & $\checkmark *$ & $\mathrm{X}$ & $\mathrm{X}$ & $\checkmark *$ & $\mathrm{X}$ & $\mathrm{X}$ & $\mathrm{X}$ \\
\hline Choi et al. [M54] & $\mathrm{X}$ & P* & $\mathrm{X}$ & $\mathrm{P}^{*}$ & $\checkmark *$ & $\sqrt{ } *$ & $\mathrm{X}$ & $\mathrm{X}$ & $\mathrm{P}$ \\
\hline Sotsenko et al. [M55] & $\mathrm{X}$ & $\mathrm{P}^{*}$ & $\checkmark$ & $\checkmark$ & $\checkmark *$ & $\mathrm{P}^{*}$ & $\mathrm{P}^{*}$ & $\mathrm{X}$ & $\mathrm{P}$ \\
\hline $\mathrm{Li}$ and Chen [M56] & $\mathrm{X}$ & $\mathrm{X}$ & $\mathrm{X}$ & $\mathrm{X}$ & $\mathrm{X}$ & $\mathrm{P}$ & $\mathrm{P}^{*}$ & $\checkmark$ & $\mathrm{X}$ \\
\hline Chellouche et al. [M57] & $\mathrm{P}$ & $\mathrm{P}^{*}$ & $\mathrm{P}$ & $\mathrm{P}$ & $\checkmark$ & $\mathrm{X}$ & $\checkmark *$ & $\mathrm{P}$ & $\mathrm{X}$ \\
\hline Zhang et al. [M58] & $\mathrm{X}$ & $\mathrm{P}^{*}$ & $\mathrm{P}^{*}$ & $\checkmark *$ & $\mathrm{X}$ & $\checkmark *$ & $\mathrm{P}^{*}$ & $\mathrm{X}$ & $\mathrm{P}$ \\
\hline Berri [M59] & $P$ & P* & $\checkmark *$ & $\checkmark$ & $\mathrm{X}$ & $\sqrt{*}$ & $\mathrm{P}^{*}$ & $\checkmark$ & $\mathrm{X}$ \\
\hline Li et al. [M60] & $\mathrm{P}$ & $\mathrm{P}^{*}$ & $\checkmark$ & $\checkmark$ & $\checkmark *$ & $\checkmark$ & $\mathrm{X}$ & $\mathrm{X}$ & $\mathrm{X}$ \\
\hline Katsumata [M61] & $\mathrm{P}$ & $\mathrm{X}$ & $\checkmark$ & $\mathrm{P}$ & $\mathrm{X}$ & $\mathrm{X}$ & $\mathrm{P}$ & $\mathrm{X}$ & $\mathrm{X}$ \\
\hline Da et al. [M62] & $\mathrm{X}$ & $\mathrm{P}$ & $\mathrm{X}$ & $\mathrm{X}$ & $\mathrm{X}$ & $\mathrm{P}$ & $\mathrm{P}^{*}$ & $\mathrm{X}$ & $\mathrm{X}$ \\
\hline Benlamri and Zhang [M63] & $\mathrm{P}$ & $\mathrm{P}^{*}$ & $\checkmark *$ & $\mathrm{X}$ & $\checkmark *$ & $\checkmark$ & $\mathrm{P}^{*}$ & $\mathrm{P}$ & $\mathrm{X}$ \\
\hline Miraoui [M64] & $\mathrm{P}$ & $\checkmark *$ & $\mathrm{X}$ & $\mathrm{X}$ & $\mathrm{X}$ & $\mathrm{X}$ & $\mathrm{P}^{*}$ & $\mathrm{P}$ & $\mathrm{X}$ \\
\hline Rahman et al. [M65] & $\mathrm{X}$ & $\mathrm{X}$ & $\checkmark *$ & $\sqrt{ } *$ & $\mathrm{X}$ & $\checkmark *$ & $\mathrm{P}^{*}$ & $\checkmark *$ & $\mathrm{X}$ \\
\hline Diallo et al. [M66] & $\mathrm{X}$ & $\checkmark$ & $\checkmark$ & $\mathrm{X}$ & $\checkmark$ & $\checkmark$ & $\mathrm{P}^{*}$ & $\mathrm{P}$ & $\mathrm{P}$ \\
\hline Maalej et al. [M67] & $\mathrm{P}$ & $\mathrm{P}^{*}$ & $\checkmark$ & $\checkmark$ & $\checkmark$ & $\checkmark$ & $\checkmark$ & $\mathrm{P}^{*}$ & $\mathrm{X}$ \\
\hline Furno and Zimeo [M68] & $\checkmark *$ & $\mathrm{X}$ & $\mathrm{X}$ & $\checkmark$ & $P$ & $\checkmark *$ & $\mathrm{P}$ & $\checkmark$ & $\mathrm{X}$ \\
\hline Novakovic and Huemer [M69] & $\mathrm{P}$ & $\mathrm{X}$ & $\checkmark *$ & $\mathrm{X}$ & $\mathrm{P}$ & $\mathrm{P}$ & P* & $\mathrm{X}$ & $\mathrm{X}$ \\
\hline Ai et al. [M70] & P* & $\mathrm{P}$ & $\mathrm{X}$ & $\checkmark *$ & P* & $\sqrt{*}$ & $\mathrm{P}^{*}$ & $\mathrm{X}$ & $\mathrm{P}$ \\
\hline Boudaa et al. [M71] & $\checkmark$ & $\mathrm{P}^{*}$ & $\checkmark$ & $\checkmark$ & $\checkmark *$ & $\checkmark$ & $\checkmark$ & $\mathrm{X}$ & $\mathrm{X}$ \\
\hline Kim et al. [M72] & P* & P* & $\checkmark *$ & $\checkmark *$ & $\mathrm{X}$ & $\mathrm{X}$ & P* & $\mathrm{X}$ & $\mathrm{P}$ \\
\hline Fissaa et al. [M73] & $\mathrm{P}$ & $\mathrm{P}^{*}$ & $\mathrm{X}$ & $\checkmark$ & $\mathrm{P}$ & $\checkmark$ & $\mathrm{P}^{*}$ & $\mathrm{P}$ & $\mathrm{P}^{*}$ \\
\hline Zouhaier et al. [M74] & $\mathrm{P}$ & $\mathrm{P}$ & $\checkmark$ & $\mathrm{X}$ & $\checkmark$ & $\mathrm{X}$ & $\mathrm{P}^{*}$ & $\mathrm{P}$ & $\mathrm{X}$ \\
\hline Kabir et al. [M75] & $\mathrm{P}$ & $\mathrm{X}$ & $\mathrm{P}$ & $\mathrm{X}$ & $\mathrm{X}$ & $\mathrm{X}$ & $\mathrm{P}^{*}$ & $\mathrm{P}$ & $\checkmark$ \\
\hline Paul.and Wachsmuth [M76] & $\mathrm{P}$ & $\mathrm{X}$ & $\checkmark$ & $\mathrm{X}$ & $\mathrm{P}$ & $\checkmark$ & $\mathrm{P}$ & $\mathrm{X}$ & $\mathrm{X}$ \\
\hline Hu et al. [M77] & $\mathrm{P}$ & $\mathrm{X}$ & $\checkmark$ & $\checkmark$ & $\mathrm{P}$ & $\checkmark$ & $\mathrm{P}^{*}$ & $\mathrm{P}$ & $\mathrm{X}$ \\
\hline Copeland and Crespi [M78] & $\mathrm{X}$ & $\mathrm{X}$ & $\checkmark$ & $\checkmark$ & $\mathrm{P}$ & $\mathrm{P}$ & $\mathrm{P}^{*}$ & $\mathrm{X}$ & $\mathrm{X}$ \\
\hline Wong et al. [M79] & $\checkmark$ & $\mathrm{P}^{*}$ & $\checkmark *$ & $\mathrm{X}$ & $\mathrm{X}$ & $\sqrt{*}$ & $\mathrm{P}^{*}$ & $\mathrm{P}$ & $\mathrm{X}$ \\
\hline Jianfeng and Dong [M80] & $\mathrm{P}^{*}$ & P* & $\mathrm{X}$ & $\checkmark *$ & $\mathrm{P}^{*}$ & $\checkmark$ & $\mathrm{P}$ & $\mathrm{X}$ & $\mathrm{X}$ \\
\hline Madkour and Maach [M81] & $\mathrm{P}$ & $\mathrm{P}^{*}$ & $\checkmark$ & $\mathrm{X}$ & $\mathrm{P}^{*}$ & $\checkmark$ & $\checkmark$ & $\mathrm{P}$ & $\mathrm{P}^{*}$ \\
\hline Jang and Choi [M82] & $\mathrm{P}$ & $\checkmark *$ & $\checkmark *$ & $\checkmark *$ & $\mathrm{P}$ & $\checkmark *$ & $\checkmark *$ & $\mathrm{X}$ & $\mathrm{X}$ \\
\hline Gilman et al. [M83] & $\mathrm{P}$ & $\mathrm{P}^{*}$ & $\mathrm{P}$ & $\checkmark$ & $\mathrm{P}^{*}$ & $\checkmark$ & $\checkmark$ & $\mathrm{P}$ & $\mathrm{P}^{*}$ \\
\hline Wusheng et al. [M84] & $\mathrm{P}^{*}$ & $\mathrm{P}$ & $\checkmark$ & $\mathrm{X}$ & $\mathrm{P}$ & $\checkmark$ & $\mathrm{P}^{*}$ & $\mathrm{P}$ & $\mathrm{P}$ \\
\hline $\mathrm{Li}[\mathrm{M} 85]$ & $\mathrm{P}^{*}$ & P* & $\checkmark *$ & $\checkmark$ & $\mathrm{P}^{*}$ & $\checkmark *$ & $\mathrm{P}^{*}$ & $\mathrm{P}$ & $\mathrm{X}$ \\
\hline Kim et al. [M86] & $\mathrm{P}^{*}$ & $\mathrm{P}$ & $\mathrm{P}$ & $\mathrm{X}$ & $\checkmark$ & $\mathrm{P}$ & $\mathrm{P}^{*}$ & $\mathrm{P}$ & $\mathrm{X}$ \\
\hline Changboka et al. [M87] & $\mathrm{X}$ & $\checkmark$ & $\checkmark$ & $\checkmark *$ & $\mathrm{X}$ & $\mathrm{X}$ & $\sqrt{*}$ & $\mathrm{P}$ & $\mathrm{X}$ \\
\hline Durán et al. [M88] & $\mathrm{P}$ & $\mathrm{X}$ & $\mathrm{X}$ & $\mathrm{X}$ & $\checkmark *$ & $\checkmark$ & $\checkmark *$ & $\mathrm{P}$ & $\checkmark$ \\
\hline Tong et al. [M89] & $\mathrm{P}$ & P* & $\mathrm{X}$ & $\mathrm{X}$ & $\mathrm{P}$ & $\checkmark$ & $\mathrm{P}^{*}$ & $\mathrm{X}$ & $\mathrm{P}$ \\
\hline Luo et al. [M90] & $\mathrm{P}$ & $\mathrm{P}$ & $\mathrm{X}$ & $\checkmark$ & $\mathrm{P}$ & $\mathrm{X}$ & $\mathrm{P}^{*}$ & $\mathrm{X}$ & $\mathrm{X}$ \\
\hline Yi et al. [M91] & $\mathrm{P}$ & P* & $\mathrm{P}$ & $\mathrm{X}$ & $\checkmark$ & $\mathrm{X}$ & P* & $\mathrm{P}$ & $\mathrm{P}$ \\
\hline Sun et al. [M92] & $\mathrm{P}$ & $\mathrm{P}^{*}$ & $\checkmark$ & $\mathrm{X}$ & $\checkmark *$ & $\mathrm{X}$ & $\mathrm{P}^{*}$ & $\mathrm{P}$ & $\mathrm{P}$ \\
\hline Sigg and David [M93] & $\mathrm{P}^{*}$ & P* & $\checkmark *$ & $\checkmark *$ & $\checkmark *$ & $\checkmark *$ & $\checkmark$ & $\checkmark$ & $\mathrm{P}^{*}$ \\
\hline Park et al. [M94] & $\mathrm{P}$ & P* & $\checkmark$ & $\mathrm{X}$ & $\mathrm{P}$ & $\checkmark$ & $\mathrm{P}^{*}$ & $\mathrm{X}$ & $\mathrm{P}$ \\
\hline Zainol and Nakata [M95] & $\mathrm{P}^{*}$ & $\mathrm{P}^{*}$ & $\checkmark$ & $\mathrm{X}$ & $\mathrm{P}^{*}$ & $\checkmark$ & $\checkmark$ & $\mathrm{X}$ & $\mathrm{P}$ \\
\hline Saeedi et al.[M96] & $\mathrm{P}$ & $\checkmark$ & $\checkmark *$ & $\mathrm{X}$ & $\mathrm{X}$ & $\checkmark *$ & $\mathrm{P}^{*}$ & $\mathrm{X}$ & $\mathrm{X}$ \\
\hline Bandara et al. [M97] & $\mathrm{X}$ & P* & $\mathrm{X}$ & $\mathrm{X}$ & $\mathrm{X}$ & $\mathrm{X}$ & $\mathrm{P}^{*}$ & $\mathrm{X}$ & $\mathrm{P}$ \\
\hline Liu et al. [M98] & $\mathrm{P}$ & $\mathrm{P}^{*}$ & $\mathrm{P}$ & $\checkmark$ & $\checkmark *$ & $\sqrt{ } *$ & $\mathrm{P}$ & $\mathrm{X}$ & $\checkmark *$ \\
\hline $\mathrm{Hu}$ and $\mathrm{Li}$ [M99] & $P$ & $\mathrm{P}^{*}$ & $\mathrm{X}$ & $\checkmark$ & $\checkmark *$ & $\checkmark$ & $\mathrm{P}^{*}$ & $\mathrm{P}^{*}$ & $\mathrm{P}^{*}$ \\
\hline Cioara et al. [M100] & $\checkmark$ & $\checkmark *$ & $\mathrm{X}$ & $\mathrm{X}$ & $\mathrm{P}$ & $\mathrm{P}$ & $\mathrm{P}^{*}$ & $\mathrm{P}$ & $\mathrm{X}$ \\
\hline Cao et al. [M101] & $\mathrm{P}^{*}$ & $\mathrm{P}^{*}$ & $\checkmark$ & $\mathrm{X}$ & $\mathrm{P}$ & $\checkmark$ & $\mathrm{P}^{*}$ & $\mathrm{X}$ & $\mathrm{P}$ \\
\hline Lamsfus et al. [M102] & $\mathrm{X}$ & $\mathrm{P}^{*}$ & $\checkmark$ & $\checkmark$ & $\mathrm{P}$ & $\checkmark$ & $\mathrm{P}^{*}$ & $\mathrm{P}$ & $\mathrm{X}$ \\
\hline
\end{tabular}




\begin{tabular}{|c|c|c|c|c|c|c|c|c|c|}
\hline Proposals & Agent & Resource & Activity & Time & $\begin{array}{c}\text { Enviro } \\
\text { nment }\end{array}$ & Location & Profile & Role & $\begin{array}{c}\text { States \& } \\
\text { Status }\end{array}$ \\
\hline Hur et al. [M103] & $\mathrm{P}$ & $\mathrm{P}$ & $\checkmark$ & $X$ & $\mathrm{P}$ & $\checkmark$ & $\checkmark$ & $\mathrm{X}$ & $\mathrm{X}$ \\
\hline Coma et al. [M104] & $\mathrm{X}$ & $\mathrm{X}$ & $\mathrm{P}$ & $\sqrt{ } *$ & $\mathrm{P}$ & $\mathrm{P}$ & $\mathrm{P}^{*}$ & $\mathrm{P}$ & $\mathrm{X}$ \\
\hline Tan et al. [M105] & $\mathrm{P}^{*}$ & $\mathrm{P}^{*}$ & $\sqrt{ } *$ & $\checkmark$ & $\checkmark *$ & $\checkmark *$ & $\checkmark$ & $\checkmark$ & $\checkmark$ \\
\hline Sun et al. [M106] & $\mathrm{P}$ & $\mathrm{P}^{*}$ & $\mathrm{P}$ & $\checkmark$ & $\sqrt{ } *$ & $\sqrt{ } *$ & $\mathrm{P}^{*}$ & $\mathrm{P}$ & $\mathrm{P} *$ \\
\hline Ouyang et al. [M107] & $\sqrt{ } *$ & $\mathrm{P}^{*}$ & $\mathrm{P}$ & $\mathrm{X}$ & $\sqrt{ } *$ & $\mathrm{X}$ & $\mathrm{P}^{*}$ & $\mathrm{P}$ & $\mathrm{X}$ \\
\hline Ko and Sim [M108] & $\mathrm{X}$ & $\mathrm{P}^{*}$ & $\checkmark *$ & $\mathrm{X}$ & $\mathrm{X}$ & $\sqrt{ } *$ & $\checkmark$ & $\mathrm{X}$ & $\mathrm{X}$ \\
\hline Chang et al. [M109] & $\mathrm{X}$ & $\mathrm{X}$ & $\mathrm{X}$ & $\mathrm{X}$ & $\mathrm{X}$ & $\mathrm{X}$ & $\mathrm{X}$ & $\mathrm{X}$ & $\sqrt{ } *$ \\
\hline Ghadiri et al. [M110] & $\mathrm{P}$ & $\mathrm{P}^{*}$ & $\mathrm{X}$ & $\checkmark$ & $\mathrm{P}$ & $\checkmark$ & $\mathrm{P}^{*}$ & $\mathrm{X}$ & $\mathrm{P} *$ \\
\hline Erfianto et al. [M111] & $\mathrm{P}^{*}$ & $\mathrm{P}^{*}$ & $\checkmark$ & $\mathrm{X}$ & $\mathrm{P}$ & $\checkmark$ & $\mathrm{P}^{*}$ & $X$ & $\mathrm{X}$ \\
\hline Qin et al. [M112] & $\mathrm{P}^{*}$ & $\mathrm{P}^{*}$ & $\checkmark *$ & $\checkmark$ & $\checkmark$ & $\checkmark$ & $\mathrm{P}$ & $\mathrm{P}$ & $\mathrm{X}$ \\
\hline Lee and Meier [M113] & $\mathrm{X}$ & $\mathrm{X}$ & $\mathrm{X}$ & $\checkmark$ & $\mathrm{P}$ & $\checkmark$ & $\mathrm{P}$ & $\mathrm{X}$ & $\mathrm{P}$ \\
\hline Mei et al. [M114] & $\mathrm{X}$ & $\checkmark$ & $\mathrm{X}$ & $\mathrm{X}$ & $\mathrm{P}$ & $\mathrm{X}$ & $\mathrm{P}$ & $\mathrm{P}$ & $\mathrm{X}$ \\
\hline Paganelli et al. [M115] & $\checkmark *$ & $\mathrm{P}^{*}$ & $\checkmark$ & $\mathrm{X}$ & $\checkmark$ & $\checkmark$ & $\mathrm{P}^{*}$ & $\mathrm{P}$ & $\mathrm{X}$ \\
\hline Hwang et al. [M116] & $\mathrm{P} *$ & $\mathrm{P}^{*}$ & $\mathrm{P}^{*}$ & $\sqrt{ } *$ & $\mathrm{P}^{*}$ & $\checkmark$ & $\checkmark$ & $\mathrm{P}^{*}$ & $\mathrm{P}$ \\
\hline Choi and Yoon [M117] & $\mathrm{P}^{*}$ & $\mathrm{P}^{*}$ & $\checkmark$ & $\checkmark$ & $\checkmark$ & $\checkmark$ & $\checkmark$ & $\mathrm{P}$ & $\mathrm{X}$ \\
\hline Hwang et al. [M118] & $\mathrm{P}$ & $X$ & $\mathrm{P}$ & $\mathrm{X}$ & $\mathrm{P}^{*}$ & $\checkmark$ & $\checkmark$ & $\mathrm{P}$ & $X$ \\
\hline Jrad et al. [M119] & $\mathrm{X}$ & $\mathrm{P}$ & $\mathrm{X}$ & $\mathrm{X}$ & $\mathrm{X}$ & $\checkmark$ & $\mathrm{P}^{*}$ & $\mathrm{X}$ & $\mathrm{P}$ \\
\hline Pessoa et al. [M120] & $\mathrm{P}$ & $\mathrm{P}^{*}$ & $\checkmark *$ & $X$ & $X$ & $\checkmark$ & $\mathrm{X}$ & $X$ & $X$ \\
\hline Hamdeh and Ma [M121] & $\mathrm{P}$ & $\mathrm{P}^{*}$ & $\sqrt{ } *$ & $\checkmark$ & $\mathrm{X}$ & $\sqrt{ } *$ & $\mathrm{X}$ & $X$ & $\mathrm{P} *$ \\
\hline Strimpakou et al. [M122] & $\checkmark$ & $\mathrm{P}^{*}$ & $\sqrt{ } *$ & $\checkmark$ & $\mathrm{X}$ & $\checkmark$ & $\mathrm{P}^{*}$ & $\mathrm{X}$ & $\mathrm{P}^{*}$ \\
\hline Sheng et al. [M123] & $\mathrm{X}$ & $\mathrm{P}$ & $\mathrm{P}$ & $\mathrm{X}$ & $\mathrm{P}^{*}$ & $\mathrm{P}$ & $\mathrm{P}^{*}$ & $\mathrm{P}$ & $\mathrm{P}$ \\
\hline Wang et al. [M124] & $\mathrm{P}$ & $\mathrm{P}^{*}$ & $\mathrm{P}$ & $\mathrm{X}$ & $\mathrm{P}$ & $\mathrm{P}$ & $\mathrm{P}$ & $\mathrm{P}$ & $\mathrm{P}$ \\
\hline Yang et al. [M125] & $\mathrm{P}^{*}$ & $\mathrm{P}^{*}$ & $\mathrm{P}$ & $\checkmark$ & $\checkmark *$ & $\sqrt{ } *$ & $\sqrt{ } *$ & $\mathrm{P}$ & $\mathrm{P} *$ \\
\hline Kranenburg et al. [M126] & $\mathrm{P}$ & $\mathrm{P}^{*}$ & $\mathrm{P}$ & $\mathrm{X}$ & $\checkmark$ & $\mathrm{P}$ & $\mathrm{P}$ & $\mathrm{P}$ & $\mathrm{P}$ \\
\hline Chaari et al. [M127] & $\mathrm{P}$ & $\checkmark$ & $\checkmark$ & $\mathrm{X}$ & $\mathrm{P}$ & $\checkmark$ & $\mathrm{P}^{*}$ & $\mathrm{X}$ & $\mathrm{X}$ \\
\hline Ou et al. [M128] & $\sqrt{ } *$ & $\mathrm{P}^{*}$ & $\sqrt{ } *$ & $\checkmark$ & $\mathrm{P}$ & $\sqrt{ } *$ & $\mathrm{P} *$ & $\mathrm{P}$ & $\mathrm{X}$ \\
\hline Almeida et al. [M129] & $\mathrm{P}$ & $\mathrm{X}$ & $\mathrm{P}$ & $\mathrm{P}$ & $\mathrm{P}$ & $\checkmark$ & $\checkmark *$ & $\mathrm{P}$ & $\mathrm{P} *$ \\
\hline Hu and Moore [M130] & $\mathrm{P}$ & $\mathrm{P}$ & $\checkmark$ & $\checkmark$ & $\mathrm{P}$ & $\mathrm{P}$ & $\mathrm{P}$ & $\checkmark$ & $\sqrt{ } *$ \\
\hline Amundsen and Eliass. [M131] & $\mathrm{X}$ & $\mathrm{P}^{*}$ & $\mathrm{X}$ & $\mathrm{X}$ & $\mathrm{X}$ & $\mathrm{X}$ & $\mathrm{P}^{*}$ & $\mathrm{X}$ & $\mathrm{P} *$ \\
\hline Hong et al. [M132] & $\mathrm{P}^{*}$ & $\mathrm{P}^{*}$ & $\checkmark$ & $\checkmark$ & $\checkmark *$ & $\sqrt{ } *$ & $\mathrm{P}$ & $\mathrm{X}$ & $\mathrm{X}$ \\
\hline Kalyan et al. [M133] & $\checkmark$ & $\mathrm{P}^{*}$ & $\mathrm{P}$ & $\checkmark$ & $\mathrm{X}$ & $\sqrt{ } *$ & $\mathrm{P}^{*}$ & $\mathrm{P}$ & $\mathrm{P}$ \\
\hline Go and Sohn [M134] & $\mathrm{P} *$ & $\mathrm{P} *$ & $\mathrm{X}$ & $\mathrm{X}$ & $\checkmark$ & $\mathrm{P}$ & $\mathrm{P}$ & $\mathrm{P}$ & $\mathrm{P}$ \\
\hline Zacarias et al. [M135] & $\mathrm{P}$ & $\mathrm{X}$ & $\mathrm{P}$ & $\checkmark$ & $\mathrm{X}$ & $\mathrm{X}$ & $\mathrm{X}$ & $\checkmark$ & $\mathrm{X}$ \\
\hline Ko et al. [M136] & $\mathrm{P}$ & $\mathrm{P} *$ & $\checkmark$ & $\mathrm{X}$ & $\checkmark *$ & $\mathrm{X}$ & $\checkmark$ & $\mathrm{X}$ & $\mathrm{P} *$ \\
\hline Kaenampor. and Neill [M137] & $\mathrm{P}^{*}$ & $\mathrm{P}^{*}$ & $\mathrm{P}$ & $\checkmark$ & $\mathrm{P}$ & $\checkmark$ & $\checkmark$ & $\checkmark$ & $\mathrm{X}$ \\
\hline Korpipaa et al. [M138] & $\mathrm{P}$ & $\mathrm{P} *$ & $\checkmark$ & $\checkmark$ & $\sqrt{*}$ & $\checkmark$ & $\mathrm{P} *$ & $\mathrm{X}$ & $\mathrm{X}$ \\
\hline$\checkmark$ & $7 \%$ & $7 \%$ & $26 \%$ & $38 \%$ & $14 \%$ & $36 \%$ & $19 \%$ & $15 \%$ & $5 \%$ \\
\hline$\sqrt{ } *$ & $12 \%$ & $8 \%$ & $22 \%$ & $12 \%$ & $17 \%$ & $29 \%$ & $7 \%$ & $1 \%$ & $4 \%$ \\
\hline $\mathbf{P}$ & $42 \%$ & $12 \%$ & $18 \%$ & $4 \%$ & $30 \%$ & $12 \%$ & $15 \%$ & $33 \%$ & $24 \%$ \\
\hline $\mathbf{P *}$ & $19 \%$ & $57 \%$ & $6 \%$ & $1 \%$ & $13 \%$ & $3 \%$ & $49 \%$ & $7 \%$ & $12 \%$ \\
\hline $\mathbf{X}$ & $20 \%$ & $17 \%$ & $28 \%$ & $46 \%$ & $27 \%$ & $20 \%$ & $10 \%$ & $43 \%$ & $55 \%$ \\
\hline
\end{tabular}

We also identified the target domain of the different proposals, since it has an influence in the proposed vocabulary. The proposals targeted 16 different domains, being the most referenced ones: 1) services in Smart Cities and the Internet of Things (28 proposals, 20.0\%) covering services in smart home, smart parking, smart campus, smart agents, public transportation, etc.; 2) software services (20 proposals, $14.5 \%$ ) covering web service composition, discovery and adaptation, service provisioning and consumption and common services (e.g., email services); 3) business processes from the point of view of services (15 proposals, 10.9\%) covering BPM, WoT business environment, business to business, etc.; 4) e-health services (15 proposals, $10.9 \%$ ) covering healthcare services, health monitoring, etc.; 5) mobile computing (12 proposals, $8.7 \%$ ) covering user interface tailored for disable users, social-aware applications, etc.; 6) generic models for services in the ubiquitous computing (9 proposals, 6.5\%) covering middleware frameworks, social context. From the 138 proposals, 64 of them $(46.4 \%)$ were generic proposals that were enlarged to demonstrate their extensibility in some domains specified. 


\subsection{RQ1.4. What are the most consolidated classes of context information and entities in ontology- based context models?}

The goal in this research question is to analyze and evaluate the provided definitions of classes of context information and entities, with the objective of providing a set of definitions semantically coherent and generic enough for grounding context knowledge with lower levels of abstraction. For this goal, we analyzed the most consolidated definitions for classes of context information and entities in the selected context models. More precisely, we focused on the first level classes, which are the most frequent ones in the surveyed context models, and their synonyms (see Table 4). Hence, in order to obtain accurate and significant results, the analysis focused on clustering definitions that represent a generic description of a set of classes that share an exact or similar meaning (synonyms) without being ambiguous or highly abstract. Next, we sought and proposed, by considering the retrieved definitions, a definition taken from WordNet ${ }^{4}$ [35] with the aim of decreasing misunderstandings of the clustered definitions, and therefore consolidating and unifying the generic view of such definitions. Our intention was to specify generic definitions that might fundament and cover several classes, rather than providing the "best" definition of classes. As an example, consider the following case of the Agent class:

- Def.1. "Both computational entities and human users can be modelled as agents" [M6];

- Def.2. "Represents the users" [M30];

- Def.3. "Agent who performs the activity" [M31];

- Def.4. "It provides information about the identities of the entities in the environment" [M32];

- WordNet. "A representative who acts on behalf of other persons or organizations" [35];

As it can be seen, the definitions follow certain similarity to each other. However, some of them are more generic than others. Using WordNet as stated above, we clarify the meaning of Agent in our ontology. From this perspective, we are able to say that an Agent can be a computational entity, human, user, etc., that performs an activity and that might acts on behalf of other agents.

Classes with larger hierarchical depth provide very specific definitions that we did not consider in this analysis. However, part of our criteria to group generic definitions was that from these definitions, specific ones could be instantiated. Given the example above, we considered that the compilation between the clustered definitions and WordNet provides a consolidated and unified vision of entities in the environment.

Table 7 summarizes the results of this research question. As it can be seen, in most of the first level classes we found at least one definition that might be classified generic enough as to include other classes and their corresponding definitions. In this sense, the classes with more number of generic definitions identified are Time and Location with 4 definitions each covering around 22 and 25 classes of other proposals, respectively, followed by Activity with 2 definitions covering 20 context classes of other proposals. Contrary to them, Profile, Role and States and status were the classes with less number of generic definitions; this means that most of the classes defined regarding to them, refer to a particular domain or describe a particular entity. The results obtained show a very low number of generic definitions considering that we have analyzed 2.756 context classes. This means that most of the context models have been applied on specific scenarios of the context-aware area from the perspective of service-oriented computing, defining context classes domain-dependent that were not linked with upper level ontologies to provide a rich semantic.

\footnotetext{
${ }^{4}$ WordNet has been used for a long time as a source of nouns, verbs, adjectives and adverbs grouped into sets of cognitive synonyms (synsets), each expressing a distinct concept.
} 
Table 7: Most consolidated definitions of classes of context information and entities

\begin{tabular}{|c|c|c|}
\hline Class & Definition & Definition coverage \\
\hline Agent & $\begin{array}{l}\text { 1) "Entities that perform some action (e.g. people, group and } \\
\text { organizations)" [M21]. } \\
\text { 2) "Provides information about the identities of the entities in the } \\
\text { environment" [M32]. } \\
\text { 3) "A representative who acts on behalf of other persons or } \\
\text { organizations" [35]. }\end{array}$ & $\begin{array}{l}\text { [M6]; [M10]; [M14]; [M17]; } \\
\text { [M23]; [M30]; [M31]; [M35]; } \\
\text { [M41]; [M100]; [M110]; } \\
\text { [M115] }\end{array}$ \\
\hline Resource & $\begin{array}{l}\text { 1) "Resources express people, services or tools that can be used in to } \\
\text { achieve a particular goal or task" [M33]. } \\
\text { 2) "Describes anything used to perform the activity. Resources needed } \\
\text { by the activity" [M31]. } \\
\text { 3) "A source of aid or support that may be drawn upon when } \\
\text { needed" [35]. }\end{array}$ & $\begin{array}{l}\text { [M35]; [M37]; [M45]; [M46]; } \\
\text { [M47]; [M64]; [M100]; } \\
\text { [M114] }\end{array}$ \\
\hline Activity & $\begin{array}{l}\text { 1) "The activity context of the agents" [M17]. } \\
\text { 2) "Actions and tasks performed by an object" [M34]. } \\
\text { 3) "Any specific behaviour" [35]. }\end{array}$ & $\begin{array}{l}\text { [M3]; [M13]; [M14]; [M15]; } \\
\text { [M22]; [M23]; [M35]; [M36]; } \\
\text { [M42]; [M43] [M63]; [M77]; } \\
\text { [M81]; [M83]; [M84]; [M94]; } \\
\text { [M111]; [M115]; [M117]; } \\
\text { [M120]; [M122] }\end{array}$ \\
\hline Time & $\begin{array}{l}\text { 1) "Involves timeline of past, present, and future. This allows for a record } \\
\text { of past context, which can be used for comparison to the current context } \\
\text { or for predicting future context" [M3]. } \\
\text { 2) "Describes temporal properties and temporal relations between } \\
\text { different events" [M7]. } \\
\text { 3) "Models information that is purely temporal in nature" [M35]. } \\
\text { 4) "Temporal concepts and properties common to any formalization of } \\
\text { time" [M6]. } \\
\text { 5) "The continuum of experience in which events pass from the future } \\
\text { through the present to the past" [35]. }\end{array}$ & $\begin{array}{l}\text { [M10]; [M11]; [M18]; [M22]; } \\
\text { [M23]; [M30]; [M34]; [M36]; } \\
\text { [M42]; [M43]; [M70]; [M71]; } \\
\text { [M98]; [M110]; [M13]; } \\
\text { [M116]; [M117]; [M122]; } \\
\text { [M133]; [M135]; [M137] }\end{array}$ \\
\hline $\begin{array}{l}\text { Environ } \\
\text {-ment }\end{array}$ & $\begin{array}{l}\text { 1) "Consists in modeling all the environmental factors" [M28]. } \\
\text { 2) "The totality of surrounding conditions" [35]. }\end{array}$ & $\begin{array}{l}\text { [M3]; [M15]; [M22]; [M40]; } \\
\text { [M42]; [M43]; [M71]; [M92]; } \\
\text { [M95]; [M98]; [M117]; } \\
\text { [M134] }\end{array}$ \\
\hline Location & $\begin{array}{l}\text { 1) "By location context, we mean a collection of dynamic knowledge that } \\
\text { describes the location of an agent" [M17]. } \\
\text { 2) "Provides information about the location of entities as identified and } \\
\text { detected by location-type sensors" [M32]. } \\
\text { 3) "A location may be described as an absolute location, meaning the } \\
\text { exact location of something, or as a relative location, meaning the } \\
\text { location of something relative to something else" [M36]. } \\
\text { 4) "Represents the abstraction of a physical location, which has two } \\
\text { subclasses including LogicalLocation and PhysicalLocation" [M37]. } \\
\text { 5) "A point or extent in space" [35]. }\end{array}$ & $\begin{array}{l}\text { [M3]; [M6]; [M7]; [M10]; } \\
\text { [M13]; [M14]; [M18]; [M23]; } \\
\text { [M30]; [M40]; [M71]; [M81]; } \\
\text { [M84]; [M88]; [M110]; } \\
\text { [M111]; [M113]; [M115]; } \\
\text { [M117]; [M119]; [M120]; } \\
\text { [M122] }\end{array}$ \\
\hline Profile & $\begin{array}{l}\text { 1) "Profile Info of entities" [M38]. } \\
\text { 2) "An outline of something" [35]. }\end{array}$ & $\begin{array}{l}\text { [M2]; [M18]; [M23]; [M29]; } \\
\text { [M39]; [M67]; [M83]; [M95]; } \\
\text { [M116]; [M117]; [M118]; } \\
\text { [M129]; [M136] }\end{array}$ \\
\hline Role & $\begin{array}{l}\text { 1) "Role of an agent can be used to characterize the intention of the } \\
\text { agent" [M17]. } \\
\text { 2) "The actions and activities assigned to or required or expected of a } \\
\text { person or group" [35]. }\end{array}$ & $\begin{array}{l}\text { [M14]; [M15]; [M22]; [M30]; } \\
\text { [M45]; [M47]; [M65]; [M75]; } \\
\text { [M130]; [M135]; [M137] }\end{array}$ \\
\hline $\begin{array}{l}\text { States } \\
\text { and } \\
\text { Status }\end{array}$ & $\begin{array}{l}\text { 1) "Status Info of entities" [M38]. } \\
\text { 2) "A state at a particular time" [35]. }\end{array}$ & $\begin{array}{l}\text { [M3]; [M18]; [M29]; [M75]; } \\
\text { [M77]; [M88]; [M98] }\end{array}$ \\
\hline
\end{tabular}




\section{Discussion}

In this section, we discuss the observations gathered when answering each research question specified in this review.

RQ1.1. What is the chronological overview of the research done so far in ontology-based context models? The distribution of papers over the years (see Fig. 2) shows that ontology-based context modeling is a lively area. In fact, if we consider the selected papers (i.e., papers that fulfilled the inclusion criteria), 2014 experienced a dramatic growth becoming even the year with more selected works in the period of this review. In our opinion, the reasons for context being of such interest for the research community are twofold. On the one hand, the great importance of context in modern service-oriented computing and software systems (smart cities, IoT, self-adaptive systems, etc.) that demand deep knowledge on context in order to deliver good solutions to the citizens. On the other hand, the absence of a standard for context modeling (the most widespread proposal, SOUPA cannot be considered as such) triggers work trying to contribute in this direction.

The analysis per provenance shows an unsurprising dominance of university papers. This observation is commonplace when surveying the state of the art based exclusively in academic papers. Still, it is remarkable that such dominance is very high. If we compare to related fields, we found similar numbers in a former SM on ontologies for quality of service [36] where the percentage of industry papers was 9\%, only slightly higher than the $5 \%$ reported in this paper. It is worth remarking that the cited SM on quality of service found also a $4 \%$ of proposals coming from organizations like W3C or OASIS, which is missing in the context field. If we compare with other SMs in software engineering, we find percentages of industry papers even greater than 30\% [37][38][39].

We also distributed the context models chronologically exploring their relationships with the aim of identifying the most influencing, influenced and consolidated contributions. The most remarkable observation from this chronological analysis is that current context models have had a limited impact on each other until now: only $8 \%$ of the proposals have influenced other proposals (only two of them, CONON and SOUPA, influenced more than 5 other approaches). Remarkably, 59\% of the proposals were formulated from scratch. Also, the transitivity analysis showed that their impact didn't propagate much to subsequent models. We consider this as an indicator that the community is still not mature enough in spite of the large amount of existing proposals. On the contrary, as a positive observation, we noticed the influence of classical ontological works in some context models proposals (e.g., FOAF, OpenCyc, DAML).

Considering such findings together, they suggest the need of development of an ontology-based context model that can act as a basis of prospective proposals and that can provide a unified and consolidated body of knowledge on context modeling in the field of service-oriented computing. Such context model should comprise context knowledge pieces from the perspective of service-oriented computing and appropriate parts of the ontologies (context-oriented or not) that were the basis of different context models found in this SM. We believe that the chronological analysis shown in this SM and summarized in Figure 4 can be of great help to this future work. For instance, it shows which proposals have been defined considering existing work, or which proposals exert more influences. For its wide adoption, industry and/or standard bodies should be involved more than currently are.

RQ1.2. What are the characteristics of the proposed ontology-based context models? As the results of this research question point out, most of the models include knowledge pieces that are introduced but not defined, neither in the paper itself nor through links to external documentation. In more detail, the correlations found among these knowledge pieces reflect deficiency of completeness, clarity, consistency, expandability, robustness and coherence. This situation was mostly observed in models with big size, i.e. models providing several classes and higher depth level, and in models that integrate or merge different ontologies. Instead, models with smaller size reflect more coherence among the knowledge pieces specified 
therein. It could be thought that this fact comes from the inherent limitation of presenting research results in the limited space of a scientific paper but as commented above, the information was not available through external links either. The findings of this research questions also show that most of the relationships among context information classes represent a basic subclass relation and only a small set of proposals provide domain-dependent relationships. Both facts together claim for a more systematic and thorough presentation of ontology-based context models as a pre-requisite for their wide adoption.

The analysis of correlations among the number $\mathrm{N}$ of context information classes (which is used as indicator of the size of the models) and the rest of entity types shows an interesting fact: while there is an approximate linear relationship among $\mathrm{N}$ and the depth levels of the hierarchy (see Fig. 5), such a relationship does not exist with the others: $79 \%$ of the models have less than 10 datatype properties and $83 \%$ of them have less than 10 object properties, in both cases regardless of the value of $N$ (which ranges from 3 to more than 40). In other words, contrary to what we expected at least in object properties, the size of the analyzed datatype and object properties is not correlated with the size of classes specified. We expected $100 \%$ of completeness of object properties at least at abstract levels of the model, i.e., the first or second level of the hierarchy from which it is possible to reason and query facts and assertions that involve lower level concepts (specific classes of context information and entities) that do not have any relation with other class. Going into the details of the papers, the main reason is that these papers usually focus on only one part of the domain model in order to develop an example, while the rest of the model remains unexplored and poorly documented, as stated in the previous paragraph.

In more detail to datatype properties, although the literature does not state the most suitable number of datatype properties to link a class to a data value, we consider that the number of datatype properties provided in the reviewed context models is too low. This fact may indicate that most of the context models were designed to support complex context-aware applications or systems that do not manage simple specifications or value restrictions, i.e., describing certain context by using properties for which the value is a XML schema datatype (e.g., describing the profile of a person as an expected triple "Person hasName xsd:String") or trigger an event given certain value restriction (e.g., if a person hasAge xsd:Int greaterThan " 18 " then the person is classified as an adult). At this respect, most of the context models employed in context-aware services supporting such type of descriptions or restrictions were those focused on profiles and preferences of the user; have reused ontologies such as FOAF; describe the location of someone/something by direct values of latitude and longitude; etc. The retrieved evidence clearly leads to conclude that most of the context information classes were not linked to data values by means of datatype properties; therefore, although this situation can be caused for modelling decisions, we observe a lack to identify datatype properties consistent to the domain.

In more detail to object properties, although a set of object properties may be related only to a class in a model as also happens with the datatype properties, this correlation was expected, i.e. the higher number of classes is, the higher the number of object properties should have been. According to common literature on ontology building [23][34][40], one of the main modeling components are the object properties that represent a type of association between concepts of the domain. However, the object properties proposed in the models are too low since most of them were developed with very low range of object properties, i.e., $82.6 \%$ of the models have presented less than 10 object properties.

We also observed that although the difference of the total number between both properties ( 743 object and 694 datatypes) is not representative, most of the assessed contributions were focused on object properties. We could identify that it is because the following reasons: some context models have been designed to characterize their properties as transitive or symmetric, and only the object properties provide this type of characteristics; reasoning engines can be able to exploit the expressivity of object properties increasing the reasoning capabilities of the ontologies; powerful inferences that can be obtained from the object properties (e.g. if certain location $\mathrm{X}$ is affected by the pollution of the environment, and a person $\mathrm{Y}$ is located at $\mathrm{X}$, then the pollution of the environment also affects the person Y); etc. Most of the object properties used in 
context-aware services are those related to the Location class (e.g., isLocatedAt, isLocatedNear, etc.), Service class (e.g., usesService, providesService), User class (e.g., hasRole, hasProfile, etc.), Time class (e.g., before, after, to, from, etc.), and so on.

Regarding the completeness of ontology-based context models, we observed that the definitions of model components are very incomplete in general. At the level of classes, only a small set of proposals (21\%) have a unique and consistent definition for more than $90 \%$ of their context information classes, and $63 \%$ of them have a completeness definition below $60 \%$. The situation does not improve if we focus on datatype and object properties, e.g. only $4 \%$ of the proposals include datatype properties at least in $80 \%$ of their classes, and only $9 \%$ of them define object properties among their classes.

All in all, we can conclude that the level of detail on the context models given by the current proposals is not optimal, hampering their adoption. In fact, going back to RQ1.1, this lack of detail can be one of the reasons for the low impact of existing context models. If we go a bit further, we observe that ontologies with a more generic scope and rich expressiveness (i.e. classes of context information duly interrelated by means of data type and object properties that consider a robust specification of their characteristics -functional, symmetric, reflexive, etc.- and in some cases also involving rules), were ontologies that although have presence and importance for the contributions in the review, were not widely reused. Instead, context modelers seem to prefer the definition of lightweight ontologies, i.e. ontologies with low level of detail considering a small set of context information classes, basic structure of the model (commonly a context hierarchy), poor characterization of data type and object properties, etc. For this reason, ontologies such as SOUPA and CONON are two of the most considered in different context modelling contributions. From our point of view, this consideration is not bad, but would be interesting that the modelers can combine both perspectives in a way that the ontology can be reused minimizing the complexity of this process of reusing. In any case, maintaining ontologies in a basic structure with basic relationships seems to be enough to operate with them.

Following the development of a context model that can act as a basis of prospective proposals and considering the findings of this RQ, a future context model can comprise or avoid the following source of knowledge: 1) Given that the most of the proposals have been created from scratch, the modeler can avoid it and justify and encourage the reuse of models as suggested by the existing building methodologies of ontologies; 2) Given that a depth level indicates the level of abstraction of a class, the modeler can identify the models that provide the most abstract or domain-dependent classes of context in order to unify the abstraction of the vocabulary; 3) Given that most of the proposals do not provide the definitions of the context knowledge pieces, the modeler should consider to increase the reusability of the model by providing this kind of details; etc.

RQ1.3. Which classes of context information and entities are the most addressed in the ontologybased context models? According to our study and after processing synonymous terms, the most addressed context information classes explicitly defined are location (appearing in 65\% of the proposals), time (50\%), activity (48\%) and environment (31\%). Their wide coverage is not surprising since obviously from the Location of an entity different kind of context information classes can be derived or deduced, as happens with Time, Activity, and Environment which allows to reason about different dimensions of time, activities performed by or around entities, as well as reasoning about environment issues that could affect positively or negatively certain entities in an implicit or explicit interaction process given a Location.

Regarding entities, we found agent (19\%) and resource (15\%) as the most frequent ones. These numbers show that surveyed papers focus more on context information itself than on the entities to which this information is bound. This fact is related to the modelling styles employed in a model. We observed that most of the designers have preferred not clearly separate the entities that are being characterized by means of context information. In this regard, some models provide a more generic scope by modelling only context information without characterizing any specific entity. Such modelling styles allowed us to identify three 
patterns of combination between entities and context information classes (what we called entity-dependent, entity-independent and context-sensitive, see Figure 10). As it was observed, the most used is the contextsensitive, which from our perception, when this modelling style employs a term to define both an entity and different types of context information classes, has the problem of data redundancy and coherence in a higher percentage than the others (e.g. classes also defined as instances or properties).

The results also indicate that other classes such as profile and role appear with less frequency than the described above. It means that a small set of contributions provide axioms to describe the application domains of these context information classes. Furthermore, most of the axioms generated through these classes were focused on the following applications domains: most of the environment classes appearing in $33 \%$ of the proposals were focused on climatological parameters, most of the $27 \%$ of the profile classes were focused on human profiles, and most of the $17 \%$ of the role classes were focused on roles of a person. It indicates that the scientific community can explore for future context models, other domains of these classes such as the social environment, different profiles and roles of resources, among others.

As it was observed, all the context information classes and entities oscillate in different degrees of definitions and further elaboration (i.e., decomposition into simpler concepts) indicating the need to standardize the vocabulary and context modelling as also happen in many other areas. At this respect, we have observed that most of the vocabulary previously assessed has been used from a domain perspective, and the resulting domain ontologies developed have not been mapped with a middle-level, upper-level or foundational ontology. Similarly, the proposals that have reused or proposed an upper or middle level ontology to map domain ontologies presented 1) no mappings to foundational ontologies or any other context model proposal; and 2) mappings and reuses of different sources that lack of unification or standardization. This is one of the reasons for what we found several inconsistencies, i.e. several variants in synonyms, hierarchies and coverage of the context knowledge pieces. In fact, in a same domain we can find several lacks of a semantic unification.

Considering the findings of this RQ, a future context model can comprise or avoid the following source of knowledge: 1) Given that three types of patterns were identified, the modeler should considering the outlined benefits and drawbacks, from our point of view the entity-independent pattern allows modeling the entities that will play a role in a scenario and therefore, characterizing their situation can be more easy and the queries to the ontology to retrieve such situation by entity should be more expressive; 2) Given that there is a big inconsistency among the vocabulary used in existing contributions of context modelling, we have pointed out synonyms and hierarchies that should be considered to avoid semantic inconsistencies in the definition of the model; 3) The matches of the classes in different levels of a hierarchy indicate that existing models should be unified to provide a consistent hierarchy and better understanding of the model, it can be useful for domain ontologies with a bottom-up approach to be mapped with abstract classes; etc.

RQ1.4. What are the most consolidated classes of context information and entities? The lack of a standard and the diversity of context modelling contributions have prompted to the diversification and redefinition of different knowledge pieces of a model. Therefore, we analyzed the generality level of the definitions provided for describing the first level classes of the surveyed context models, particularly the most frequent ones. The results indicate that more than $60 \%$ of the contributions provide definitions entitydependent or domain-dependent to describe a class (context or entity) that in most of the models belongs to the first level classes. It means that the scope of these classes that can be considered generics is reduced and therefore, the expandability of a model is affected. From this perspective, the Profile class is one of the classes in which most of its definitions depend on the domain or entity related. Instead, classes such as Location, Time and Activity were defined in a more generic form, i.e. most of their definitions were not related to a domain or specific entity. Thus, a class with these features should be more easily expanded.

Finally, a future context model should consider the most consolidated definitions of classes of context information and entities to unify the heterogeneous definitions of classes, from abstract levels toward 
domain levels all of them maintaining a consistent definition and therefore, increasing the reusability capabilities of either, the entire model or the context knowledge pieces specified.

To sum up, this mapping study has pointed out that there is a big lack in reusability, standardization and consolidation of context knowledge pieces for context modeling. We identified different inconsistencies regarding context information classes and entities, one of the most important issues to reuse vocabulary of the context models analyzed is the lack of homogeneity among the classes, the resources provided and shortage of definitions. Furthermore, as it happens in many other areas, it does not exist a single context model agreed by the scientific community; instead several proposals have been presented for specific purposes. These results show the need to consolidate the context knowledge already provided. As a first step in this direction, we have made the exercise of defining the classes of context information and entities obtained from the research questions 1.3 and 1.4. Figure 11 shows the resulting context taxonomy, corresponding to a high level hierarchy view of the most addressed classes in the surveyed context models. Note that we are not providing a complete class taxonomy, but a basic taxonomy of high-level context classes feasible for extending and reusing the model. We consider that the structure provided (organized according to the entity-independent pattern, see Figure 9-B) is more generic and allows characterizing any entity with different context information classes. Hence, in this case, the surroundings of resources and agents can be modelled using the context information classes of the taxonomy. An extended report of classes (context information and entities) can be found in online Annex L at [17].

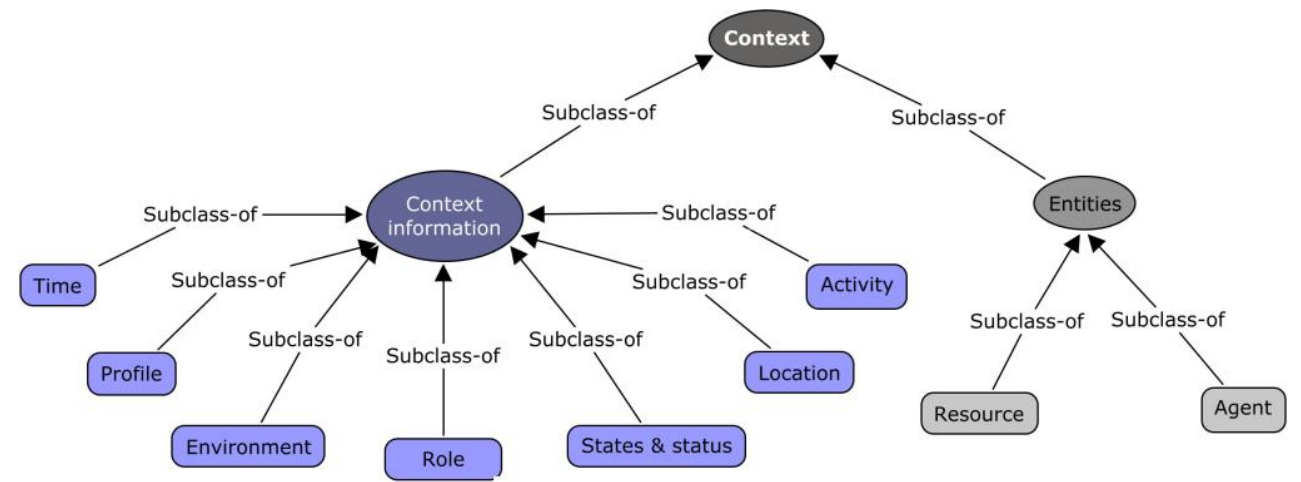

Figure 11: Basic taxonomy of high level classes that should contain a context model

\section{Threats to validity}

This section addresses both the aspects of the research process that might represent threats to validity and the actions performed to mitigate those risks. To do so, we have identified and evaluated validity threats following the common classification of validity concerns: construct validity, internal validity, external validity and conclusion validity, discussed in [41] and covered as follows:

\section{- Construct validity.}

Risks: 1) One of the inherent threats in any systematic review is that it does not guarantee the inclusion of all relevant works in the field. This represents a risk that might be caused by different reasons, such as: contributions in the field are not indexed on the selected database, the keywords defined in title and abstract of a contribution do not match with the keywords established in the search string, and so on. 2) Threats beyond an accurate protocol comprising issues related to the paper, for instance, inaccurate abstracts.

Mitigation actions: 1) We combined automatic and manual searches, considering different databases (Scopus, IEEE Xplore and ACM DL), specifying several relevant journals and conferences in the field, and studying accurately the keywords to use in the search. The identification of some basic sources (see online 
Annex $C$ at [17]) was useful since some of the conferences and journals that we considered as usual venues for the topic addressed in this review, had at least one edition not indexed in the selected databases, so that, we performed manual searches. It is worth remarking that, according to our experience, the number of papers in 2015 (and even 2014) may slightly increase in the future since digital libraries are not always up to date. However, we remind that the most critical venues have been manually checked until 2015, so that the missing papers should not be many and their relevance, limited. 2) We included a final step of snowballing, as described in Section 3.3.3, assuring the inclusion of context models that have had a biggest impact in the field.

\section{- Internal validity.}

Risks: 1) Several context models do not provide an accurate definition of the presented context information classes and entities. As shown in Section 4.2, some definitions are ambiguous, inconsistent or simply absent. This situation represents a big challenge when analyzing the coverage of context classes, and a subsequent threat to validity. 2) Given the lack of a reference context model (formal standard) and considering that each context model provides its own hierarchical structure (see Figure 10), we decided grouping synonyms analyzing each term of the proposals, verify matches and positions of each term grouped, and consolidate the information retrieved. This process can be considered by itself a threat to validity.

Mitigation actions: 1) We analyzed the list of ill-defined context classes, establishing the following strategy: for those classes lacking of a definition, if there was a clear consensus of the definition in the state of the art, or the hierarchical path was self-explanatory (e.g. if the root of profile is a user class, profile obviously refers to context information class of a user), we recognized the meaning of the context class despite the lack of a definition; classes whose definition was ambiguous or inconsistent, but it didn't affect the categorization of context classes, they were considered in the analysis, otherwise, they were discarded. Following this criterion, around 60 context classes were discarded from more than 2000 classes analyzed, representing only the $3 \%$ of this quantity. 2) To mitigate this risk, the process and specified criteria to address it were discussed and analyzed closely by all three authors of the paper, consolidating in a systematic way the retrieved context knowledge.

\section{- External validity.}

Risks: 1) Several context models were developed for specific purposes, delimiting their reusability in different cases and therefore forcing the creation of new context models starting from scratch.

Actions: 1) The service perspective allowed to consider different perspectives such as user, provider, consumer, etc. from which several context models were built. Therefore, the resources consolidated encompass all the perspectives already provided in context modeling, especially, resources regarding context information vocabulary, object and datatype properties and definitions completeness. These resources are intended to be a knowledge source easy to reuse in different use cases.

\section{- Conclusion validity.}

Risks: 1) Several surveys did not provide the guidelines addressed in the research, which limits to reproduce the research performed and therefore, the results can be different.

Actions: 1) In this sense, we have explicitly described all the steps performed in the systematic mapping by detailing the procedure as defined in the systematic mapping protocol. Furthermore, the list of papers found and selected on each step is included in online Annexes A and F at [17]. 


\section{Conclusions}

Maintaining adequate service levels plays an important role in the service-oriented computing for the comfort of users and customers. In this regard, different aspects are responsible for maintaining the required levels and to avoid risks, being the context information one of the most important aspects to be considered in the health of services and other entities that conforms the value structure.

Given the broad context applicability in different domains and perspectives and its diversity, in this survey paper was presented a systematic mapping study on context modeling focused on analyzing and evaluating pieces of context knowledge including classes (context information and entities), properties and functions. The survey has the aim of consolidating the information retrieved and provide the needed background to build or reuse context models. For this purpose, we designed and followed a review protocol that has allowed gathering 138 proposals to answer the research questions that we define as part of this process. Full details of the protocol are available at [18] as a series of online annexes.

We consider that the results of this review can be useful in the development of future context models more consolidated and in some way more standardized by considering all the semantic factors and completeness of the context resources so far provided such as classes of context information and entities, datatype and object properties, etc. making available a model easy to reuse in any contextual domain.

The main reasons for formulating a consistent proposal given the perspective and findings of this study are summarized as follows:

1) Clarity: compiling all the semantic definitions and representations of the primitives proposed in the analyzed contributions, we identified what so hard can be finding equivalences among these primitives. Some of them are too brief or too abstract, so much that, delimit their meaning and representation in other models. At this respect, we consider that the context-aware characterization of a service needs the capture of a clear and concise context model easy to be comprehensive;

2) Generality: most of the analyzed contributions were focused on the needs of an application domain providing domain-dependent ontologies and primitives hardly to reuse in new domains. At this respect, we consider that a model should provide the feasibility and facility for adding new context knowledge. Furthermore, the development of context-aware services needs the capture of the generic view of a context model (upper or middle level ontologies) for the creation of prospective services;

3) Uniformity: most of the analyzed contributions proposed ontologies without mappings to, and equivalences with, a foundational ontology, decreasing thus the semantic quality, coherent vocabulary, reusability and interoperability of proposed models. The reasons are the time consuming and operational cost when finding a common knowledge and getting acquainted with foundational ontologies. However, we consider that these costs can be balanced by increasing the sharing of knowledge and the interoperability between context-aware services when the model is grounded by foundational ontologies;

4) Explicit representability: we identified that the ontologies that define primitives (concepts and properties) without an explicit representation between these primitives and their related semantics (definitions, scope and specific relationships) considerably reduce their reasoning capabilities and therefore, also their reusability and capability of sharing knowledge between services. Hence, we consider that a model should explicitly define and represent such primitives and semantics;

5) Ambiguity: the study showed the existence of a set of inconsistencies in a model (e.g., classes also specified as properties and instances). Such issue can be a general worry of developers for reusing inconsistent models, since its main effect is also over the reasoning capabilities of the ontology. For instance, due to the noise inserted in a context model, the assertions by reasoning can produce different inconsistencies (e.g., an entity can be located in two places, a service can be asserted also as a project, etc.). Hence, we consider that the models should be subjected to different validations such as satisfying competence questions, identify inconsistencies by means of automatic tools, etc.; 
6) Modularity: the findings of the study showed that the ontologies with a modular perspective were the most ones reused by other ontologies (e.g., CONON and SOUPA). It seems that putting all the concepts together without boundaries, make barriers emerge and increases the likelihood of not reusing an ontology. Given the modularity of our proposal, the developers can reuse only the modules in which they are interested and therefore, reduce the processing time and operational costs of the ontology.

Conducting the designing of a consistent model with all the previous features seems to be so complicated, thus, developers of context-aware services still have the challenge of building consistent context models that can be considered as benchmark in the context-aware computing facilitating tasks of capturing, managing and distributing context. Concretely, a well-defined context model can contribute in tasks of configuration of physical or logical monitors, understanding the monitored data, establishment of new techniques for supporting the context life cycle, discover and selection of services, etc.

As a final conclusion, we believe that this panoramic view on the anatomy of context models may be a good reference for prospective researchers and practitioners on the field, especially with the aim of avoiding new classifications or definitions that may be contrary to the established practices that we have found.

As future work, we plan to extend the basic taxonomy depicted in Figure 11, to provide a context model aiming to standardize and consolidate a body of context knowledge that can be easily reused, extended and adapted for specific or generic purposes. Also we plan to propose a context-aware monitoring architecture for supporting the context acquisition by interacting with the context model, and therefore, supporting the whole context life cycle.

\section{Acknowledgment}

This work is partially supported by the Spanish project TIN2016-79269-R and the SUPERSEDE project, funded by the European Union's Information and Communication Technologies Programme (H2020) under grant agreement no 644018.

\section{References}

[1] Jong-yi Hong, Eui-ho Suh, and Sung-Jin Kim. 2009. Context-aware systems: a literature review and classification. Expert Systems with Applications 36, 4 (2009), 8509-8522.

[2] Joëlle Coutaz, James Crowley, Simon Dobson, and David Garlan. 2005. Context is key. Communications of the ACM 48, 3 (2005), 49-53.

[3] Charith Perera, Arkady Zaslavsky, Peter Christen, and Dimitrios Georgakopoulos. 2014. Context aware computing for the internet of things: a survey. IEEE Communications Surveys \& Tutorials 16, 1 (2014), 414-454.

[4] Kai Petersen, Sairam Vakkalanka, and Ludwik Kuzniarz. 2015. Guidelines for conducting systematic mapping studies in software engineering: an update. Information and Software Technology 64, 0 (2015), 1-18.

[5] Barbara Kitchenham, and Stuart Charters. 2007. Guidelines for performing systematic literature reviews in software engineering. Technical Report EBSE-2007-01. Keele and Durham Universities, UK.

[6] Mary Bazire, and Patrick Brézillon. 2005. Understanding context before using it. In: Modelling and Using Context. Springer-Verlag, 29-40.

[7] Anind Dey. 2001. Understanding and using context. Personal and Ubiquitous Computing 5, 1 (2001), 4-7.

[8] Elarbi Badidi, and Ikbal Taleb. 2011. Towards a cloud-based framework for context management. In: Innovations in Information Technology. IEEE, 35-40.

[9] Karen Henricksen. 2003. A framework for context-aware pervasive computing applications. Ph.D. Dissertation. Queensland University, Brisbane, Queensland, Australia.

[10] Yiwei Cao, Ralf Klamma, Min Hou, and Matthias Jarke. 2008. Follow me, follow you-spatiotemporal community context modelling and adaptation for mobile information systems. In: Mobile Data Management. IEEE, 108-115.

[11] James Crowley, Joëlle Coutaz, Gaeten Rey, and Patrick Reignier. 2002. Perceptual components for context aware computing. In: Ubiquitous Computing. ACM, 117-134.

[12] Claudio Bettini, Oliver Brdiczka, Karen Henricksen, Jadwiga Indulska, Daniela Nicklas, Anand Ranganathan, and Daniele Riboni. 2010. A survey of context modelling and reasoning techniques. Pervasive and Mobile Computing 6, 2 
(2010), 161-180.

[13] Natalya Noy, and Deborah McGuinness. 2001. Ontology development 101: a guide to creating your first ontology. Technical Report KSL-01-05. Stanford University, EEUU.

[14] Natalya Noy. 2004. Semantic integration: a survey of ontology-based approaches. ACM Sigmod Record 33, 4 (2004), 6570 .

[15] Harry Chen, Filip Perich, Tim Finin, and Anupam Joshi. 2004. SOUPA: standard ontology for ubiquitous and pervasive applications. In: Mobile and Ubiquitous Systems: Computing, Networking, and Services. Springer-Verlag, 258-267.

[16] Kalla Sudhana, Cyril Raj, and RM Suresh. 2013. An ontology-based framework for context-aware adaptive e-learning system. In: Computer Communication and Informatics. IEEE, 1-6.

[17] Oscar Cabrera, Xavier Franch, and Jordi Marco. 2016. Online annexes of ontology-based context modelling in serviceoriented computing: a systematic mapping. http://gessi.lsi.upc.edu/qcontextmodels/

[18] Christine Bauer. 2012. A comparison and validation of 13 context meta-models. In: Information Systems. AISeL, 17.

[19] Barbara Kitchenham, Pearl Brereton, Mark Turner, Mahmood Niazi, Stephen Linkman, Rialette Pretorius, and David Budgen. 2009. The impact of limited search procedures for systematic literature reviews-a participant-observer case study. In: Empirical Software Engineering and Measurement. IEEE, 336-345.

[20] Oscar Dieste, Anna Grimán, and Natalia Juristo. 2009. Developing search strategies for detecting relevant experiments. Empirical Software Engineering 14, 5 (2009), 513-539.

[21] Kai Petersen, Robert Feldt, Shahid Mujtaba, and Michael Mattsson. 2008. Systematic mapping studies in software engineering. In: Evaluation and Assessment in Software Engineering. ACM, 68-77.

[22] Barbara Kitchenham, Emilia Mendes, and Guilherme Travassos. 2007. Cross versus within-company cost estimation studies: a systematic review. IEEE Transactions on Software Engineering 33, 5 (2007), 316-329.

[23] Mariano Fernández-López, Asunción Gómez-Pérez, and Natalia Juristo. 1997. METHONTOLOGY: from ontological art towards ontological engineering. Technical Report SS-97-06. Stanford University, EEUU.

[24] Abraham Maslow, Robert Frager, and Ruth Cox. 1970. Motivation and personality (3rd ed.). James Fadiman and Cynthia McReynolds (Eds.), Vol. 2. Harper \& Row, New York.

[25] Mike Uschold, Martin King, Stuart Moralee, and Yannis Zorgios. 1998. The enterprise ontology. The Knowledge Engineering Review 13, 1 (1998), 31-89.

[26] Douglas Lenat, and Ramanathan Guha. 1989. Building large knowledge-based systems; representation and inference in the Cyc Project (1st ed.). Addison Wesley Longman Publishing Co., Inc., Boston.

[27] Albrecht Schmidt, Kofi Aidoo, Antti Takaluoma, Urpo Tuomela, Kristof Laerhoven, and Walter de Velde. 1999a. Advanced interaction in context. In: Handheld and Ubiquitous Computing. Springer-Verlag, 89-101.

[28] Albrecht Schmidt, Michael Beigl, and Hans-W. Gellersen. 1999b. There is more to context than location. Computers \& Graphics 23, 6 (1999), 893-901.

[29] Bill Schilit, Norman Adams, and Roy Want. 1994. Context-aware computing applications. In: Mobile Computing Systems and Applications. IEEE, 85-90.

[30] Feng Pan, and Jerry Hobbs. 2004. Time in owl-s. Technical Report SS-04-06. Stanford University, EEUU.

[31] Adam Pease, and Ian Niles. 2002. IEEE standard upper ontology: a progress report. The Knowledge Engineering Review 17, 1 (2002), 65-70.

[32] Dan Brickley, and Libby Miller. 2005. FOAF vocabulary specification. Technical Report ILRT. Bristol University, UK.

[33] Oscar Corcho, Mariano Fernández-López, Asunción Gómez-Pérez, and Angel López-Cima. 2005. Building legal ontologies with METHONTOLOGY and WebODE. In: Law and the Semantic Web. Springer-Verlag, 142-157.

[34] Mike Uschold, and Michael Gruninger. 1996. Ontologies: principles, methods and applications. The Knowledge Engineering Review 11, 2 (1996), 93-136.

[35] George Miller. 1995. WordNet: a lexical database for English. Communications of the ACM 38, 11 (1995), 39-41.

[36] Marc Oriol, Jordi Marco, and Xavier Franch. 2014. Quality models for web services: a systematic mapping. Information and Software Technology 56, 10 (2014), 1167-1182.

[37] David Ameller, Xavier Burgués, Oriol Collell, Dolors Costal, Xavier Franch, and Mike P. Papazoglou. 2015. Development of service-oriented architectures using model-driven development: a mapping study. Information and Software Technology 62, 0 (2015), 42-66.

[38] Darja Šmite, Claes Wohlin, Tony Gorschek, and Robert Feldt. 2010. Empirical evidence in global software engineering: a systematic review. Empirical Software Engineering 15, 1 (2010), 91-118.

[39] Everton Cavalcante, Jorge Pereira, Marcelo Alves, Pedro Maia, Roniceli Moura, Thais Batista, Flavia Delicato, and Paulo Pires. 2016. On the interplay of internet of things and cloud computing: a systematic mapping study. Computer Communications 89, 0 (2016), 17-33.

[40] Michael Uschold, and Martin King. 1995. Towards a methodology for building ontologies. In: International Joint Conference on Artificial Intelligence, Workshop on Basic Ontological Issues in Knowledge Sharing, 6.1-6.10.

[41] Claes Wohlin, Per Runeson, Martin Höst, Magnus Ohlsson, Björn Regnell, and Anders Wesslén. 2012. Experimentation in software engineering. Springer-Verlag, 9-72. 